\title{
Three-Dimensional Nonlinear Vibration Model and Fatigue Failure Mechanism of Deep-Water Test Pipe
}

\section{Xiaoqiang Guo}

Southwest Petroleum University

Yuxin Nie

Southwest Petroleum University

Jun Liu (D 201031010081@swpu.edu.cn )

Southwest Petroleum University

Yufa He

CNOOC Research Institute Co., Ltd.

Liangjie Mao

Southwest Petroleum University

\section{Guorong Wang}

Southwest Petroleum University

Liming Dai

University of Regina

\section{Research Article}

Keywords: Riser-test pipe system, Hamilton variational principle, Nonlinear vibration model, Simulation experiment, Fatigue failure mechanism

Posted Date: September 13th, 2021

DOI: https://doi.org/10.21203/rs.3.rs-866437/v1

License: (c) (i) This work is licensed under a Creative Commons Attribution 4.0 International License.

Read Full License 


\title{
Three-Dimensional Nonlinear Vibration Model and Fatigue Failure Mechanism of Deep-Water Test Pipe
}

\author{
Xiaoqiang Guo ${ }^{1,2,5}$, Yuxin $\mathrm{Nie}^{1}$, Jun $\mathrm{Liu}^{1,2^{*}}$, Yufa $\mathrm{He}^{3,4}$, Liangjie $\mathrm{Mao}^{3}$, Guorong Wang ${ }^{1,3}$, Liming Dai ${ }^{5}$ \\ ${ }^{I}$ School of Mechatronic Engineering, Southwest Petroleum University, Chengdu, 610500, China \\ ${ }^{2}$ School of Mechanical Engineering, Chengdu University, Chengdu, 610106, China. \\ ${ }^{3}$ Energy Equipment Institute, Southwest Petroleum University, Chengdu, 610500, China \\ ${ }^{4}$ CNOOC Research Institute Co., Ltd., Beijing 100028, China \\ ${ }^{5}$ Industrial Systems Engineering, University of Regina, Regina, SK S4S 0A2, Canada. \\ *Corresponding author, Email: 201031010081@swpu.edu.cn (J. Liu)
}

\begin{abstract}
In deep-water test conditions, the riser-test pipe system (RTS) is subject to the vortex induced effect on riser, flow induced effect on test pipe and longitudinal/transverse coupling effect, which is prone to buckling deformation, fatigue fracture and friction perforation. To resolve this, the three-dimensional (3D) nonlinear vibration model of deep-water RTS is established using the micro-finite method, energy method and Hamilton variational principle. Based on the elastic-plastic contact collision theory, the nonlinear contact load calculation method between riser and test pipe is proposed. Compared with experimental measurement results, calculation results of the proposed vibration model in this study and the single tubing vibration model in our recent work, the correctness and effectiveness of the proposed vibration model of the deep-water RTS are verified. Meanwhile, the cumulative damage theory is used to establish the fatigue life prediction method of test pipe. Based on that, the influences of outflow velocity, internal flow velocity, significant wave height, as well as top tension coefficient on the fatigue life of test pipe are systematically analyzed. The results demonstrate that, first, with the increase of outflow velocity, the maximum alternating stress, the annual fatigue damage rate increased and the service life decreased significantly. The locations where fatigue failure of the test tube is easy to occur are mainly distributed at the upper "one third" and the bottom of test pipe. Second, with the increase of internal flow velocity, the "one third effect" of the test pipe will decrease, and is shown "the bottom damage effect", which needs the attention of field operators. Third, during field operation, it is necessary to properly configure the top tension coefficient so that there can be a certain relaxation between the riser and the test pipe, so as to cause transverse vibration and consume some axial energy and load. The study led to the formulation of a theoretical method for safety evaluation and a practical approach for effectively improving the fatigue life of deep-water test pipe.
\end{abstract}

Keywords: Riser-test pipe system; Hamilton variational principle; Nonlinear vibration model; Simulation experiment; Fatigue failure mechanism 


\section{Introduction}

With the increasing demand for oil\&gas resources in the world, the exploitation trend of offshore oil\&gas resources gradually develops from shallow water (Water depth is less than $500 \mathrm{~m}$ ) to deep water (Water depth is between $500 \mathrm{~m}$ and $1500 \mathrm{~m}$ ). The riser-test pipe system (RTS) is the core equipment for deep-water oil\&gas exploitation, but which is the weakest equipment. Compared with conventional water depth testing conditions, the RTS is subjected to greater risks in deep-water test conditions; these risks are mainly caused by severe non-periodic vibrations of the riser and test pipe (RTS) induced by the vortex induced effect on riser, flow induced effect on test pipe, nonlinear contact/collision of the tube in tube and longitudinal/transverse coupling effect, thereby making the RTS more susceptible to buckling deformation (Fig. 1(a)), fatigue fracture(Fig. 1(b)) and friction perforation (Fig. 1(c)) [1]. Once the system structure is damaged, it will lead to serious offshore oil\&gas accidents, resulting in significant economic losses and environmental pollution. Therefore, the three-dimensional (3D) nonlinear vibration model and fatigue failure mechanism for deep-water RTS should be investigated.

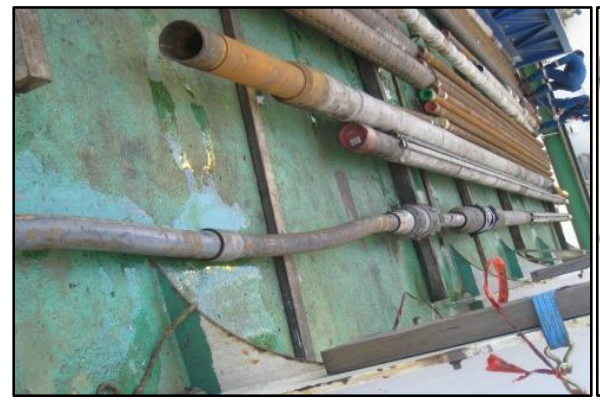

(a) Buckling deformation

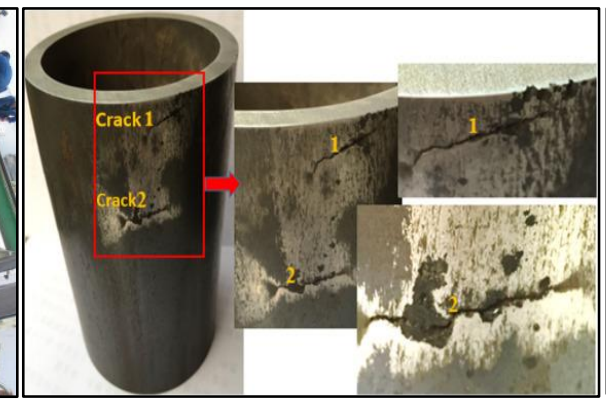

(b) Fatigue fracture

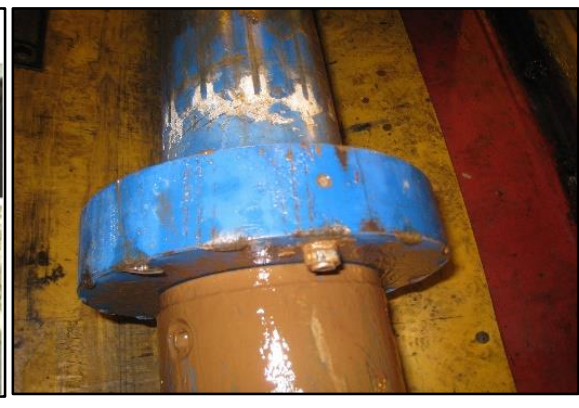

(c) Friction perforation

Fig. 1. Failure forms of the riser-test pipe system (RTS)

In early vortex-induced vibration (VIV) studies, most of the work focused on rigid cylinders [2, 3], in which the general VIV mechanism and law, such as the frequency-locked phenomenon [4] and lagging behavior [5]. In recent years, driven by offshore oil\&gas exploitation, more and more attention has been paid to the VIV problem of flexible cylinders in which the aspect ratio is a very important parameter. Physical experiments [6-8] and computational fluid dynamics (CFD) numerical simulations [9-12] are the two most common methods in these studies, and remarkable progress was made. However, when the aspect ratio of a cylinder is large or a solid model is used, physical experiments usually become very expensive and impractical, and CFD numerical simulation is too time-consuming and difficult. Therefore, in the VIV study of risers, there are relatively few works that consider a large aspect ratio (the ratio of length to diameter is greater than 1000) or the actual size. In addition to a large aspect ratio, the impact of the ocean environment load on the VIV behavior of a riser is significant. The VIV response mechanism of a flexible riser under shear flow was examined by Mathelin et al. [13] using a wake oscillator model presented by Facchinetti et al. [5]. Since the VIV amplitude in the crossflow (CF) direction is larger than that in the inline (IL) direction, most work has focused on the VIV in the CF direction [14]. The effects of the flow velocity, top tension, and pipe diameter on VIV 
behavior in the crossflow direction of a riser were studied by Xu et al. [15] and He et al. [16] using a VIV model. In the above studies, the VIV behavior in the IL direction and its influence were not taken into account. However, it was found in the work of Jauvtis and Williamson [17] that as the mass ratio $m^{*}\left(=4 m_{s} / \rho \pi D^{2}\right)$, the ratio of the structural quality to the mass of discharged fluid was less than 6.0, and the IL vibration of a cylinder could not be neglected. The VIV characteristics study of a rigid cylinder presented by Gu et al. [18], Martins et al. [19] and Gao et al. [20] also showed that the effect of the IL vibration was significant. In our recent work [21, 22], the response characteristics of VIV of marine risers with consideration of the coupling effects of the CF and IL vibration were investigated. It was found that the frequency locking effect in the uniform flow and the multi frequency effect in the shear flow for the IL vibration.

The deep-water test pipe and tubing string represent the same kind of tubular structure. The deep-water test pipe is the name of the tubular structure under the test condition, and the tubing string is the name of the tubular structure under the production condition. Therefore, their vibration characteristics have the same trend, which are nonlinear vibration induced by inside flow. Aiming at the flow induced vibration of tubular structure; firstly, some scholars [23-24] have verified the phenomenon that the internal fluid can induce the vibration of the tubular structure through experiments, which points out the direction for the later scholars' research. However, the calculation method of interaction force and the vibration model of the tubular structure are not obtained. Subsequently, many scholars carried out detailed research on the calculation method of interaction force and the vibration model, and established the calculation method of fluid force [25], the string vertical vibration [26, 27], the lateral vibration [28, 29], and the fluid-structure coupled vibration model $[30,31]$. However, the above models mainly focus on the vibration in a single direction, which can effectively predict the vibration of tubular structure with small aspect ratio, and its calculation accuracy decreased to predict the vibration of tubular structure with large aspect ratio. Also, some scholars [32-34] have found that the longitudinal/lateral coupling effects of tubular structure with large aspect ratio cannot be ignored. Therefore, in our recent work [21, 22], the longitudinal/lateral coupling model of marine risers with consideration of the coupling effects of the CF and IL vibration were established. In the actual operation process, there are other structures outside the tubing string to restrict its vibration, resulting in the structure of pipe in pipe. Therefore, the contact/collision between pipes is also one of the important factors in tubing string modeling. This research has also attracted the interest of some scholars and carried out corresponding research. In which, the bracing effect of the outer pipe was taken into account by some researchers $[35,36]$ to analyze the static buckling deformation of a tubing string. Meanwhile, the commercial software were used by researchers $[37,38]$ to investigate the impact force/friction force in the flow-induced vibration of slender structures in vertical well. Also, in our recent work [39, 40], the flowinduced nonlinear vibration model of tubing string in conventional oil\&gas wells was established, which considers the longitudinal/lateral coupling effect of tubing string and the nonlinear contact collision effect of tubing-casing. In summary, the interaction between riser and test pipe is ignored in the above studies, 
which make the calculation results by the single vibration model are not in accordance with the actual. Especially in the deep water test condition, with the increase of length diameter ratio, the interaction between riser and test pipe cannot be ignored.

The vibration failure of tubular structure has been paid attention to by scholars, mainly including wear failure, fatigue failure and insufficient strength failure [41]. In this work, the vibration fatigue failure of deep-water RTS is mainly studied. Therefore, the current research on fatigue failure of tubular structure investigated and analyzed. Lekkala et al. [42] established a fatigue life prediction model of riser using SHEAR7 commercial software, and provides the new optimized excitation coefficient datasets, which reduce the error in the predicting the VIV response of the riser. Chen et al. [43] analyzed root cause of tubing and casing failures in low-temperature carbon dioxide injection well, and an optimal tubing-casing combination is proposed to prolong the operation life of tubing. Moreover, in view of the fatigue failure of the risers caused by VIV, the researchers have established a fatigue life prediction method for risers in deep-water $[44,45]$. However, in their method, the acquisition of alternating stress ignores the contact and collision factors between riser and test pipe, which makes it impossible to accurately simulate the fatigue life of riser under severe working conditions.

In this study, the 3D nonlinear vibration model of deep-water RTS is established. The Lagrange and cubic Hermite functions are used to discretize the governing equations. Then, the incremental form of Newmark- $\beta$ and Newton-Raphson are used to solve the 3D nonlinear vibration model. Meanwhile, a vibration test bench for the RTS is designed using similarity principle, and the correctness and effectiveness of the proposed 3D nonlinear vibration model are verified by comparing with experimental data. Meanwhile, the cumulative damage theory is used to establish the fatigue life prediction method of test pipe combined with the stress response which was determined by the proposed model and the S-N curve of the pipe material (13Cr-L80) which was measured by fatigue test. Finally, the influences of outflow velocity, internal flow velocity, significant wave height, as well as top tension coefficient on the fatigue life of test pipe are systematically analyzed.

\section{3D nonlinear vibration model of the RTS}

\subsection{Nonlinear vibration control equation of the RTS}

In this section, the 3D vibration control equations of infinitesimal riser-test pipe (RTS) were established through the energy method and Hamilton variational principle. Owing to the infinitesimal segment of the RTS is very short, which can be regarded as a straight segment. Therefore, a coordinate system is established in which the depth direction set as $z$-axis, the horizontal direction (the IL direction) set as $x$-axis and the $y$-axis (the CF direction) satisfies the right-hand rule (Fig. 2). The following basic assumptions are made before modeling.

(1) The material mechanical property of riser and test pipe is ideal isotropic and elastic. 
(2) The gravity and frictional resistance are evenly distributed on the tubing element.

(3) The test pipe axis is coincided with the riser axis at initial moment, and the gravity of the RTS acts on itself at initial moment.

(4) The friction coefficient at each location of the system is constant.

(1) Vibration control equation of riser

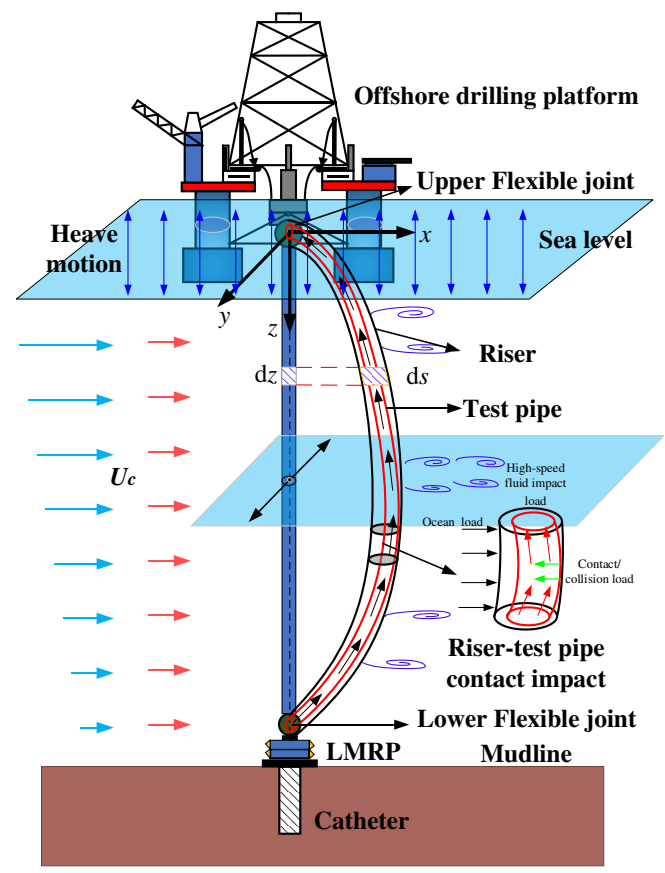

Fig. 2. Structure diagram of the RTS

Based on the small deformation hypothesis and the Kirchhoff hypothesis [22], the three displacement field components $u_{1}, u_{2}$ and $u_{3}$ along the coordinates $x, y$ and $z$, respectively, can be written as:

$$
\left\{\begin{array}{l}
u_{1}(z, t)=v_{x}(z, t), u_{2}(z, t)=v_{y}(z, t) \\
u_{3}(z, x, t)=v_{z}(z, t)-x \frac{\partial v_{x}(z, t)}{\partial z}-y \frac{\partial v_{y}(z, t)}{\partial z}
\end{array}\right.
$$

where $v_{x}, v_{y}$, and $v_{z}$ represent the displacement components of any section in the riser on the three coordinate axes, respectively (m).

According to Zhao's work [46], the Green strain can be expressed as follows:

$$
\left\{\begin{array}{l}
\varepsilon_{x x}=\frac{\partial u_{1}}{\partial x}, \quad \varepsilon_{y y}=\frac{\partial u_{2}}{\partial y}, \quad \varepsilon_{z z}=\frac{\partial u_{3}}{\partial z}+\frac{1}{2}\left(\left(\frac{\partial u_{1}}{\partial z}\right)^{2}+\left(\frac{\partial u_{2}}{\partial z}\right)^{2}\right) \\
\varepsilon_{x z}=\frac{1}{2}\left(\frac{\partial u_{1}}{\partial z}+\frac{\partial u_{3}}{\partial x}\right), \varepsilon_{y z}=\frac{1}{2}\left(\frac{\partial u_{2}}{\partial z}+\frac{\partial u_{3}}{\partial y}\right) \varepsilon_{x y}=\frac{1}{2}\left(\frac{\partial u_{1}}{\partial y}+\frac{\partial u_{2}}{\partial x}\right)
\end{array}\right.
$$

Substituting the Eq. (1) into the Eq. (2), the following equation can thus be obtained:

$$
\left\{\begin{array}{l}
\varepsilon_{z z}=\frac{\partial v_{z}}{\partial z}-x \frac{\partial^{2} v_{x}}{\partial z^{2}}-y \frac{\partial^{2} v_{y}}{\partial z^{2}}+\frac{1}{2}\left(\left(\frac{\partial v_{x}}{\partial z}\right)^{2}+\left(\frac{\partial v_{y}}{\partial z}\right)^{2}\right) \\
\varepsilon_{x x}=\varepsilon_{y y}=\varepsilon_{x z}=\varepsilon_{y z}=\varepsilon_{x y}=0
\end{array}\right.
$$

Based on the second Kirchhoff stress and neglecting Poisson effect, Hooke's law under uniaxial stress 
state is written as:

$$
\sigma_{z z}=E \varepsilon_{z z}
$$

Since the infinitesimal segment of the tubing is a standard cylinder, the integral satisfies the following formula:

$$
\int_{A} x \mathrm{~d} A=\int_{A} y \mathrm{~d} A=\int_{A} x y \mathrm{~d} A=0, \int_{A} x^{2} \mathrm{~d} A=\int_{A} y^{2} \mathrm{~d} A=I
$$

According to elastic-plastic mechanics [47] and Eq. (1) Eq. (5), the total kinetic energy $T$, potential energy $U$ and energy with external force $W$ of the riser can be respectively expressed as:

$$
\left\{\begin{array}{l}
T=\int_{0}^{L} \int_{A} \frac{\rho_{v}}{2}\left(\dot{u}_{1}^{2}+\dot{u}_{2}^{2}+\dot{u}_{3}^{2}\right) \mathrm{d} A \mathrm{~d} z=\frac{1}{2} \int_{0}^{L}\left[m_{v}\left(\dot{v}_{x}^{2}+\dot{v}_{y}^{2}+\dot{v}_{z}^{2}\right)+\rho_{v} I_{v}\left(\dot{v}_{x}^{\prime 2}+\dot{v}_{y}^{\prime 2}\right)\right] \mathrm{d} z \\
U=\frac{1}{2} \int_{V} \sigma \varepsilon \mathrm{d} V=\frac{E}{2} \int_{V} \varepsilon_{z z}^{2} \mathrm{~d} V=\frac{1}{2} \int_{0}^{L}\left[E A_{v}\left(v_{z}^{\prime}+\frac{1}{2}\left(v_{x}^{\prime 2}+v_{y}^{\prime 2}\right)\right)^{2}+E I_{v}\left(v_{x}^{\prime \prime 2}+v_{y}^{\prime \prime 2}\right)\right] \mathrm{d} z \\
W=\int_{0}^{L}\left[\left(F_{D}(z, t)+F_{x}(z, t)-c_{v} \dot{v}_{x}\right) \mathrm{d} v_{x}+\left(F_{L}(z, t)+F_{y}(z, t)-c_{v} \dot{v}_{y}\right) \mathrm{d} v_{y}+\left(F_{z}(z, t)+w_{g}-c_{v} \dot{v}_{z}\right) \mathrm{d} v_{z}\right] \mathrm{d} z
\end{array}\right.
$$

where $E$ is the elastic modulus of the riser and test pipe $(\mathrm{Pa}) ; A_{v}$ is the cross-sectional area of the riser $\left(\mathrm{m}^{2}\right) ; v_{i}^{\prime}, i=x, y, z, v_{i}^{\prime \prime}, i=x, y, z$ are the first-order and second derivative of riser displacements versus $z$, respectively; $\dot{v}_{i}, i=x, y, z$ are the first-order derivative of riser displacements versus time in $x$-, $y$ - and $z$ directions $(\mathrm{m} / \mathrm{s}) ; m_{v}$ is the mass of the per unit length riser $(\mathrm{kg}) ; \rho_{v}$ is the density of the riser $\left(\mathrm{kg} / \mathrm{m}^{3}\right)$; $I_{v}$ is the polar moment of inertia of the riser $\left(\mathrm{m}^{4}\right) ; F_{x}(z, t), F_{y}(z, t), F_{z}(z, t)$ are the contact/impact and friction force of riser-test pipe in $x$-, $y$ - and $z$-directions $(\mathrm{N})$, which can be determined in the Section 2.2; $F_{D}(z, t), F_{L}(z, t)$ are the drag force in the IL direction and the lateral lift in the CF direction $(\mathrm{N})$, which also can be determined in the Section 2.2; $c_{v}\left(=2 m_{v} \omega_{v} \zeta\right)$ is the structural damping coefficient of riser $\left(\omega_{v}\right.$ is natural angular frequency of riser, $\omega_{v}=\pi^{2} \sqrt{E I_{v} /\left(m_{v} L_{v}^{4}\right)}, L_{v}$ is the length of riser $(\mathrm{m}) ; \zeta$ is structural damping ratio); and $w_{g}\left(=m_{v} g-\rho_{w} \pi D_{o}^{2} / 4\right)$ is buoyant weight of riser per unit length (N) ( $\rho_{w}$ the density of the sea-water $\left(\mathrm{kg} / \mathrm{m}^{3}\right) ; D_{o}$ is the riser outer diameter $(\mathrm{m})$ ).

For a riser section with length $\mathrm{d} z$, its length $\mathrm{d} s$ after deformation can be written as follows:

$$
\mathrm{d} s=\sqrt{1+v_{x}^{\prime 2}+v_{y}^{\prime 2}} \mathrm{~d} z
$$

Therefore, the longitudinal strain and its variation of the riser can be written respectively as

$$
v_{z}^{\prime}=\varepsilon_{0}+\frac{\mathrm{d} s-\mathrm{d} z}{\mathrm{~d} z} \cong \varepsilon_{0}+\frac{1}{2}\left(v_{x}^{\prime 2}+v_{y}^{\prime 2}\right) \Rightarrow \delta v_{z}^{\prime}=v_{x}^{\prime} \delta v_{x}^{\prime}+v_{y}^{\prime} \delta v_{y}^{\prime}
$$

According to variational principle $\left(\left.\delta U\right|_{t=t_{1,2} / x=0, \mathrm{~L}}=\left.\delta U^{\prime}\right|_{t=t_{1,2} / x=0, \mathrm{~L}}=\left.\delta \dot{U}\right|_{t=t_{1,2} / x=0, \mathrm{~L}}=0\right)$ and integration by parts $\left(\int f^{\prime}(z) g(z) \mathrm{d} z=f(z) g(z)-\int f(z) g^{\prime}(z) \mathrm{d} z\right)$, the integration forms of $T, U$ and $W$ over time 
can be expressed as:

$$
\begin{aligned}
& \delta \int_{t_{1}}^{t_{2}} T \mathrm{~d} t=\frac{1}{2} \delta \int_{t_{1}}^{t_{2}} \int_{0}^{L}\left[m_{v}\left(\dot{v}_{x}^{2}+\dot{v}_{y}^{2}+\dot{v}_{z}^{2}\right)+\rho_{v} I_{v}\left(\dot{v}_{x}^{\prime 2}+\dot{v}_{y}^{\prime 2}\right)\right] \mathrm{d} z \mathrm{~d} t \\
& =\int_{t_{1}}^{t_{2}} \int_{0}^{L}\left[m_{v}\left(\dot{v}_{x} \delta \dot{v}_{x}+\dot{v}_{y} \delta \dot{v}_{y}+\dot{v}_{z} \delta \dot{v}_{z}\right)+\rho_{v} I_{v}\left(\dot{v}_{x}^{\prime} \delta \dot{v}_{x}^{\prime}+\dot{v}_{y}^{\prime} \delta \dot{v}_{y}^{\prime}\right)\right] \mathrm{d} z \mathrm{~d} t \\
& =\int_{0}^{L}\left[\int_{t_{1}}^{t_{2}} m_{v}\left(\dot{v}_{x} \mathrm{~d} \delta v_{x}+\dot{v}_{y} \mathrm{~d} \delta v_{y}+\dot{v}_{z} \mathrm{~d} \delta v_{z}\right)+\rho_{v} I_{v}\left(\dot{v}_{x}^{\prime} \mathrm{d} \delta v_{x}^{\prime}+\dot{v}_{y}^{\prime} \mathrm{d} \delta v_{y}^{\prime}\right)\right] \mathrm{d} z \\
& =\int_{0}^{L}\left[\begin{array}{l}
m_{v}\left(\left.\left(\dot{v}_{z} \delta v_{z}+\dot{v}_{z} \delta v_{z}+\dot{v}_{z} \delta v_{z}\right)\right|_{t_{1}} ^{t_{2}}-\int_{t_{1}}^{t_{2}}\left(\ddot{v}_{x} \delta v_{x}+\ddot{v}_{y} \delta v_{y}+\ddot{v}_{z} \delta v_{z}\right) \mathrm{d} t\right) \\
+\rho_{v} I_{v}\left(\left.\left(\dot{v}_{x}^{\prime} \delta v_{x}^{\prime}+\dot{v}_{y}^{\prime} \delta v_{y}^{\prime}\right)\right|_{t_{1}} ^{t_{2}}-\int_{t_{1}}^{t_{2}}\left(\ddot{v}_{x}^{\prime} \delta v_{x}^{\prime}+\ddot{v}_{y}^{\prime} \delta v_{y}^{\prime}\right) \mathrm{d} t\right)
\end{array}\right] \mathrm{d} z \\
& =-\int_{0}^{L} \int_{t_{1}}^{t_{2}} m_{v}\left(\ddot{v}_{x} \delta v_{x}+\ddot{v}_{y} \delta v_{y}+\ddot{v}_{z} \delta v_{z}\right) \mathrm{d} t \mathrm{~d} z-\rho_{v} I_{v} \int_{t_{1}}^{t_{2}} \int_{0}^{L}\left(\ddot{v}_{x}^{\prime} \mathrm{d} \delta v_{x}+\ddot{v}_{y}^{\prime} \mathrm{d} \delta v_{y}\right) \mathrm{d} t \\
& =-\int_{0}^{L} \int_{t_{1}}^{t_{2}} m_{v}\left(\ddot{v}_{x} \delta v_{x}+\ddot{v}_{y} \delta v_{y}+\ddot{v}_{z} \delta v_{z}\right) \mathrm{d} t \mathrm{~d} z-\rho_{v} I_{v} \int_{t_{1}}^{t_{2}}\left[\left.\left(\ddot{v}_{x}^{\prime} \delta v_{x}+\ddot{v}_{y}^{\prime} \delta v_{y}\right)\right|_{0} ^{L}-\int_{0}^{L}\left(\ddot{v}_{x}^{\prime \prime} \delta v_{x}+\ddot{v}_{y}^{\prime \prime} \delta v_{y}\right) \mathrm{d} z\right] \mathrm{d} t \\
& =\int_{t_{1}}^{t_{2}} \int_{0}^{L}\left[-m_{v}\left(\ddot{v}_{x} \delta v_{x}+\ddot{v}_{y} \delta v_{y}+\ddot{v}_{z} \delta v_{z}\right)+\rho_{v} I_{v}\left(\ddot{v}_{x}^{\prime \prime} \delta v_{x}+\ddot{v}_{y}^{\prime \prime} \delta v_{y}\right)\right] \mathrm{d} z \mathrm{~d} t \\
& \delta \int_{t_{1}}^{t_{2}} U \mathrm{~d} t=\frac{1}{2} \delta \int_{t_{1}}^{t_{2}} \int_{0}^{L}\left[E A_{v}\left(v_{z}^{\prime}+\frac{1}{2}\left(v_{x}^{\prime 2}+v_{y}^{\prime 2}\right)\right)^{2}+E I_{v}\left(v_{x}^{\prime \prime 2}+v_{y}^{\prime \prime 2}\right)\right] \mathrm{d} z \mathrm{~d} t \\
& =\int_{t_{1}}^{t_{2}} \int_{0}^{L}\left[E A_{v}\left(v_{z}^{\prime}+\frac{1}{2}\left(v_{x}^{\prime 2}+v_{y}^{\prime 2}\right)\right)\left(\delta v_{z}^{\prime}+v_{x}^{\prime} \delta v_{x}^{\prime}+v_{y}^{\prime} \delta v_{y}^{\prime}\right)+E I_{v}\left(v_{x}^{\prime \prime} \delta v_{x}^{\prime \prime}+v_{y}^{\prime \prime} \delta v_{y}^{\prime \prime}\right)\right] \mathrm{d} z \mathrm{~d} t \\
& =E A_{v} \int_{t_{1}}^{t_{2}} \int_{0}^{L}\left[\left(v_{z}^{\prime}+\frac{1}{2}\left(v_{x}^{\prime 2}+v_{y}^{\prime 2}\right)\right)\left(\mathrm{d} \delta v_{z}+v_{x}^{\prime} \mathrm{d} \delta v_{x}+v_{y}^{\prime} \mathrm{d} \delta v_{y}\right)\right] \mathrm{d} t+E I_{v} \int_{t_{1}}^{t_{2}} \int_{0}^{L}\left[\left(v_{x}^{\prime \prime} \mathrm{d} \delta v_{x}^{\prime}+v_{y}^{\prime \prime} \mathrm{d} \delta v_{y}^{\prime}\right)\right] \mathrm{d} t \\
& \left.=E A_{v} \int_{t_{1}}^{t_{2}}\left\{\left[\begin{array}{l}
\left(v_{z}^{\prime}+\frac{1}{2}\left(v_{x}^{\prime 2}+v_{y}^{\prime 2}\right)\right) \cdot \\
\left(\delta v_{z}+v_{x}^{\prime} \delta v_{x}+v_{y}^{\prime} \delta v_{y}\right)
\end{array}\right] \|_{0}^{L}-\int_{0}^{L}\left[\begin{array}{l}
\left(v_{z}^{\prime}+\frac{1}{2}\left(v_{x}^{\prime 2}+v_{y}^{\prime 2}\right)\right)^{\prime} \delta v_{z}+ \\
{\left[\left(v_{z}^{\prime}+\frac{1}{2}\left(v_{x}^{\prime 2}+v_{y}^{\prime 2}\right)\right) v_{x}^{\prime}\right]^{\prime}+\left[\left(v_{z}^{\prime}+\frac{1}{2}\left(v_{x}^{\prime 2}+v_{y}^{\prime 2}\right)\right) v_{y}^{\prime}\right.}
\end{array}\right]\right] \mathrm{d} z\right\} \mathrm{d} t \\
& +E I_{v} \int_{t_{1}}^{t_{2}}\left\{\left.\left(v_{x}^{\prime \prime} \delta v_{x}^{\prime}+v_{y}^{\prime \prime} \delta v_{y}^{\prime}\right)\right|_{0} ^{L}-\int_{0}^{L}\left[\left(v_{x}^{\prime \prime}\right)^{\prime} \mathrm{d} \delta v_{x}+\left(v_{y}^{\prime \prime}\right)^{\prime} \mathrm{d} \delta v_{y}\right]\right\} \mathrm{d} t \\
& =-E A_{v} \int_{t_{1}}^{t_{2}} \int_{0}^{L}\left[\left(v_{z}^{\prime}+\frac{1}{2}\left(v_{x}^{\prime 2}+v_{y}^{\prime 2}\right)\right)^{\prime} \delta v_{z}+\left[\left(v_{z}^{\prime}+\frac{1}{2}\left(v_{x}^{\prime 2}+v_{y}^{\prime 2}\right)\right) v_{x}^{\prime}\right]^{\prime}+\left[\left(v_{z}^{\prime}+\frac{1}{2}\left(v_{x}^{\prime 2}+v_{y}^{\prime 2}\right)\right) v_{y}^{\prime}\right]^{\prime}\right] \mathrm{d} z \mathrm{~d} t \\
& +E I_{v} \int_{t_{1}}^{t_{2}}\left\{\left.\left(v_{x}^{\prime \prime} \delta v_{x}^{\prime}+v_{y}^{\prime \prime} \delta v_{y}^{\prime}\right)\right|_{0} ^{L}-\left.\left[\left(v_{x}^{\prime \prime}\right)^{\prime} \mathrm{d} \delta v_{x}+\left(v_{y}^{\prime \prime}\right)^{\prime} \mathrm{d} \delta v_{y}\right]\right|_{0} ^{L}+\int_{0}^{L}\left[\left(v_{x}^{\prime \prime}\right)^{\prime \prime} \delta v_{x}+\left(v_{y}^{\prime \prime}\right)^{\prime \prime} \delta v_{y}\right] \mathrm{d} z\right\} \mathrm{d} t \\
& =-E A_{v} \int_{t_{1}}^{t_{2}} \int_{0}^{L}\left[\left(v_{z}^{\prime}+\frac{1}{2}\left(v_{x}^{\prime 2}+v_{y}^{\prime 2}\right)\right)^{\prime} \delta v_{z}+\left[\left(v_{z}^{\prime}+\frac{1}{2}\left(v_{x}^{\prime 2}+v_{y}^{\prime 2}\right)\right) v_{x}^{\prime}\right]^{\prime}+\left[\left(v_{z}^{\prime}+\frac{1}{2}\left(v_{x}^{\prime 2}+v_{y}^{\prime 2}\right)\right) v_{y}^{\prime}\right]^{\prime}\right] \mathrm{d} z \mathrm{~d} t \\
& +E I_{v} \int_{t_{1}}^{t_{2}} \int_{0}^{L}\left[\left(v_{x}^{\prime \prime}\right)^{\prime \prime} \delta v_{x}+\left(v_{y}^{\prime \prime}\right)^{\prime \prime} \delta v_{y}\right] \mathrm{d} z \mathrm{~d} t \\
& \delta \int_{t_{1}}^{t_{2}} W \mathrm{~d} t=\int_{t_{1}}^{t_{2}} \int_{0}^{L}\left[\left(\begin{array}{l}
F_{D}(z, t)+ \\
F_{x}(z, t)-c \dot{v}_{x}
\end{array}\right) \delta v_{x}+\left(\begin{array}{l}
F_{L}(z, t)+ \\
F_{y}(z, t)-c \dot{v}_{y}
\end{array}\right) \delta v_{y}+\left(\begin{array}{l}
F_{z}(z, t)+ \\
w_{g}-c \dot{v}_{z}
\end{array}\right) \delta v_{z}\right] \mathrm{d} z \mathrm{~d} t
\end{aligned}
$$

Substituting Eq. (9) Eq. (11) into the expression of Hamiltonian principle $\delta \int_{t_{1}}^{t_{2}}(U-T+W) \mathrm{d} t=0$,

7 
the nonlinear vibration control equations of the riser can be obtained and expressed as follows:

$$
\left\{\begin{array}{l}
m_{v} \ddot{v}_{x}+c \dot{v}_{x}-\rho_{v} I_{v} \ddot{v}_{x}^{\prime \prime}-E A_{v}\left(\left(v_{z}^{\prime}+\frac{1}{2}\left(v_{x}^{\prime 2}+v_{y}^{\prime 2}\right)\right) v_{x}^{\prime}\right)^{\prime}+E I_{v}\left(v_{x}^{\prime \prime}\right)^{\prime \prime}=F_{D}(z, t)+F_{x}(z, t) \\
m_{v} \ddot{v}_{y}+c \dot{v}_{y}-\rho_{v} I_{v} \ddot{v}_{y}^{\prime \prime}-E A_{v}\left(\left(v_{z}^{\prime}+\frac{1}{2}\left(v_{x}^{\prime 2}+v_{y}^{\prime 2}\right)\right) v_{y}^{\prime}\right)^{\prime}+E I_{v}\left(v_{y}^{\prime \prime}\right)^{\prime \prime}=F_{L}(z, t)+F_{y}(z, t) \\
m_{v} \ddot{v}_{z}+c \dot{v}_{z}-E A\left(v_{z}^{\prime \prime}+\frac{1}{2}\left(v_{x}^{\prime 2}+v_{y}^{\prime 2}\right)^{\prime}\right)=F_{z}(z, t)+w_{g}
\end{array}\right.
$$

(2) Vibration control equation of test pipe

In the oil\&gas test condition, the test pipe is located inside the riser, forming a pipe in pipe structure, and there exist high-speed test oil\&gas in the test pipe. Therefore, the main factors causing the nonlinear vibration of the test pipe include the contact/collision of the external riser and the impact of the internal high-speed oil\&gas. In this study, the same coordinate system as riser was established. Based on the assumption of Eq. (1) and Green's strain, the expressions of strain energy, kinetic energy and work done can be obtained as follows, and the kinetic energy of the fluid in the pipe was considered.

$$
\left\{\begin{array}{l}
\bar{T}=\frac{1}{2} \int_{0}^{L}\left[m_{s}\left(\dot{S}_{x}^{2}+\dot{S}_{y}^{2}+\dot{S}_{z}^{2}\right)+\rho_{s} I_{s} \cdot\left(\dot{S}_{x}^{\prime 2}+\dot{S}_{y}^{\prime 2}\right)+m_{i}\left(v_{x}^{2}+v_{y}^{2}+v_{z}^{2}\right)\right] \mathrm{d} z \\
\bar{U}=\frac{1}{2} \int_{0}^{L}\left[E A_{s}\left(S_{z}^{\prime}+\frac{1}{2}\left(S_{x}^{\prime 2}+S_{y}^{\prime 2}\right)\right)^{2}+E I_{s}\left(S_{x}^{\prime \prime 2}+S_{y}^{\prime \prime 2}\right)\right] \mathrm{d} z \\
\bar{W}=\int_{0}^{L}\left(\left(\begin{array}{l}
F_{x}(x, t)+ \\
f_{x}(x, t)-c_{s} \dot{S}_{x}
\end{array}\right) \mathrm{d} S_{x}+\left(\begin{array}{l}
F_{y}(x, t)+ \\
f_{y}(x, t)-c_{s} \dot{S}_{y}
\end{array}\right) \mathrm{d} S_{y}+\left(\begin{array}{l}
F_{z}(x, t)+f_{z}(x, t) \\
+w_{s}-c_{s} \dot{S}_{z}
\end{array}\right) \mathrm{d} S_{z}\right) \mathrm{d} z
\end{array}\right.
$$

where $S_{x}, S_{y}$, and $S_{z}$ are the displacement components of any section in the test pipe on the three coordinate axes, respectively (m). $m_{s}$ and $m_{i}$ are the masses of the per unit length test pipe and the gas per unit length $(\mathrm{kg})$, respectively; $A_{s}$ is the cross-sectional area of the test pipe $\left(\mathrm{m}^{2}\right) ; S_{i}^{\prime}, i=x, y, z$ and $S_{i}^{\prime \prime}, i=x, y, z$ are the first-order and second derivative of test pipe displacements versus $z$, respectively; $\dot{S}_{i}, i=x, y, z$ are the first-order derivative of test pipe displacements versus time in $x$-, $y$ - and $z$-directions $(\mathrm{m} / \mathrm{s}) ; f_{x}(z, t), f_{y}(z, t), f_{z}(z, t)$ are the high-speed fluid impact load in test pipe in $x$-, $y$ - and $z$ directions $(\mathrm{N})$, which also can be determined in the Section 2.2; $c_{s}\left(=2 m_{s} \omega_{s} \zeta\right)$ is the structural damping coefficient of test pipe $\left(\omega_{s}\right.$ is natural angular frequency of test pipe); and $w_{s}\left(=m_{s} g\right)$ is weight of test pipe per unit length $(\mathrm{N}) ; v_{x}, v_{y}$, and $v_{z}$ represent the absolute velocities of the internal high-speed fluid in $x$, $y$ and $z$ directions $(\mathrm{m} / \mathrm{s})$, respectively; which can be expressed as follows:

$$
v_{x}=\dot{S}_{x}+V S_{x}^{\prime}, v_{y}=\dot{S}_{y}+V S_{y}^{\prime}, v_{z}=V
$$

where $V$ is the fluid flow velocity in the test pipe $(\mathrm{m} / \mathrm{s})$. Also, substituting Eqs. (13) (14) into the 
expression of Hamiltonian principle, the nonlinear vibration control equations of test pipe can be expressed as follows:

$$
\left\{\begin{array}{l}
\left(m_{s}+m_{i}\right) \ddot{S}_{x}-\rho_{s} I_{s} \ddot{S}_{x}^{\prime \prime}+2 m_{i} V \dot{S}_{x}^{\prime}+c_{s} \dot{S}_{x}+m_{i} V^{2} S_{x}^{\prime \prime}+E I_{s}\left(S_{x}^{\prime \prime}\right)^{\prime \prime}-E A\left(\left(S_{z}^{\prime}+\frac{1}{2}\left(S_{x}^{\prime 2}+S_{y}^{\prime 2}\right)\right) S_{x}^{\prime}\right)^{\prime}=F_{x}(z, t)+f_{x}(x, t) \\
\left(m_{s}+m_{i}\right) \ddot{S}_{y}-\rho_{s} I_{s} \ddot{S}_{y}^{\prime \prime}+2 m_{i} V \dot{S}_{y}^{\prime}+c_{s} \dot{S}_{y}+m_{i} V^{2} S_{y}^{\prime \prime}+E I_{s}\left(S_{y}^{\prime \prime}\right)^{\prime \prime}-E A\left(\left(S_{z}^{\prime}+\frac{1}{2}\left(S_{x}^{\prime 2}+S_{y}^{\prime 2}\right)\right) S_{y}^{\prime}\right)^{\prime}=F_{y}(z, t)+f_{y}(x, t)(15) \\
m_{s} \ddot{S}_{z}+c_{s} \dot{S}_{z}-E A\left(S_{z}^{\prime \prime}+\frac{1}{2}\left(S_{x}^{\prime 2}+S_{y}^{\prime 2}\right)^{\prime}\right)=F_{z}(z, t)+f_{z}(x, t)+w_{s}
\end{array}\right.
$$

\subsection{Boundary conditions}

In the deep-water test condition, the external loads on the RTS include the ocean environment load and the high-speed fluid impact load, and the internal loads are the nonlinear contact collision between the riser and test pipe. The upper part of the RTS is connected with the drilling platform, and the lower part is connected with the blowout preventer (BOP). Thus, the upper displacement boundary considers the heave motion of the platform, and the lower displacement boundary is hinged support. In this section, the calculation methods of ocean environment load, contact force between riser and test pipe, impact load of high-speed fluid in pipe, and heave motion equation of platform will be established.

(1) Ocean environment load (load boundary condition)

It is assumed that the relative velocity between the fluid and the riser is $V_{r}$, and the outflow velocity of the riser is $U_{c}$. For steady flow, the steady-state drag force and lift force acting on the riser are shown in Fig. 3.
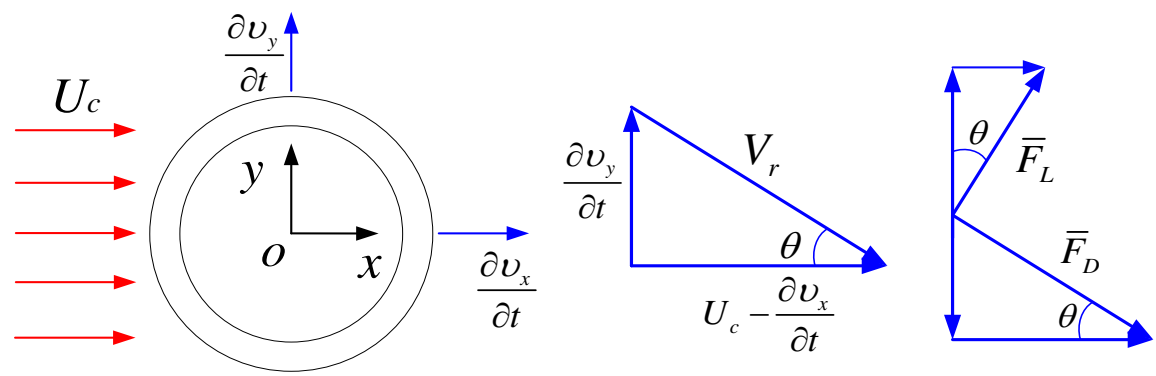

Fig. 3. External fluid forces acting on the riser

According to Morison's equation, the steady-state drag and lift forces acting on the riser is written as:

$$
\left\{\begin{array}{l}
\bar{F}_{D}=\frac{1}{2} \rho_{w} V_{r}^{2} D_{o} \bar{C}_{d} \\
\bar{F}_{L}=\frac{1}{2} \rho_{w} V_{r}^{2} D_{o} \bar{C}_{l}
\end{array}\right.
$$

where $\bar{C}_{d}$ is the coefficient of steady-state drag force, $\bar{C}_{l}$ is coefficient of steady lift force and $\rho_{w}$ is sea-water density $\left(\mathrm{kg} / \mathrm{m}^{3}\right)$.

The components of fluid force acting on the riser in the IL direction and CF direction can be expressed as follows: 


$$
\left\{\begin{array}{l}
F_{x}^{\prime}=\frac{1}{2} \rho_{w} V_{r}^{2} D_{o}\left(\bar{C}_{d} \cos \theta+\bar{C}_{l} \sin \theta\right) \\
F_{y}^{\prime}=\frac{1}{2} \rho_{w} V_{r}^{2} D_{o}\left(-\bar{C}_{d} \sin \theta+\bar{C}_{l} \cos \theta\right)
\end{array}\right.
$$

Given that

$$
\left\{\begin{array}{l}
\theta=\tan ^{-1}\left(\frac{\partial v_{y}}{\partial t} /\left(U_{c}-\frac{\partial v_{x}}{\partial t}\right)\right) \\
V_{r}=\sqrt{\left(\frac{\partial v_{y}}{\partial t}\right)^{2}+\left(U_{c}-\frac{\partial v_{x}}{\partial t}\right)^{2}}
\end{array}\right.
$$

The components of fluid force can be written further as:

$$
\left\{\begin{array}{l}
F_{x}^{\prime}=\frac{1}{2} \rho_{w} U_{c} D_{o}\left[\bar{C}_{d}\left(V_{r}-\frac{V_{r}}{U_{c}} \frac{\partial v_{x}}{\partial t}\right)+\bar{C}_{l} \frac{V_{r}}{U_{c}} \frac{\partial v_{y}}{\partial t}\right] \\
F_{y}^{\prime}=\frac{1}{2} \rho_{w} U_{c} D_{o}\left[\bar{C}_{l}\left(V_{r}-\frac{V_{r}}{U} \frac{\partial v_{x}}{\partial t}\right)-\bar{C}_{d} \frac{V_{r}}{U_{c}} \frac{\partial v_{y}}{\partial t}\right]
\end{array}\right.
$$

In addition to the steady-state component, the fluid force acting on the riser also includes harmonic pulsating drag force and pulsating lift force.

$$
\left\{\begin{array}{l}
F_{D}^{\prime}=\frac{1}{2} \rho_{w} U_{c}^{2} D_{o} C_{D} \\
F_{L}^{\prime}=\frac{1}{2} \rho_{w} U_{c}^{2} D_{o} C_{L}
\end{array}\right.
$$

where $F_{D}{ }^{\prime}, C_{D}$ respectively denote the fluctuating drag force and corresponding coefficient; $F_{L}{ }^{\prime}, C_{L}$ respectively denote fluctuating lift force and corresponding coefficient.

Therefore, according to Eqs. (19) and (20), all fluid forces acting on the riser can be written as:

$$
\left\{\begin{array}{l}
F_{D}(z, t)=F_{x}+F_{D}^{\prime}=\frac{1}{2} \rho_{w} U_{c} D_{o}\left[\bar{C}_{d}\left(V_{r}-\frac{V_{r}}{U_{c}} \frac{\partial v_{x}}{\partial t}\right)+\bar{C}_{l} \frac{V_{r}}{U_{c}} \frac{\partial v_{y}}{\partial t}\right]+\frac{1}{2} \rho_{w} U_{c}^{2} D_{o} C_{D} \\
F_{L}(z, t)=F_{y}+F_{L}^{\prime}=\frac{1}{2} \rho_{w} U_{c} D_{o}\left[\bar{C}_{l}\left(V_{r}-\frac{V_{r}}{U} \frac{\partial v_{x}}{\partial t}\right)-\bar{C}_{d} \frac{V_{r}}{U_{c}} \frac{\partial v_{y}}{\partial t}\right]+\frac{1}{2} \rho_{w} U_{c}^{2} D_{o} C_{L}
\end{array}\right.
$$

It is noted that the steady-state lift coefficient $\bar{C}_{l}$ is taken as 0 for the circular section and $V_{r} \approx U_{c}-\partial v_{x} / \partial t$. Ignoring the effect of higher-order terms, fluid forces can be further written as

$$
\left\{\begin{array}{l}
F_{D}(z, t)=\frac{1}{2} \rho_{w} U_{c}^{2} D_{o} \bar{C}_{d}-\rho_{w} U_{c} D_{o} \bar{C}_{d} \frac{\partial v_{x}}{\partial t}+\frac{1}{2} \rho_{w} U_{c}^{2} D_{o} C_{D} \\
F_{L}(z, t)=-\frac{1}{2} \rho_{w} U_{c} D_{o} \bar{C}_{d} \frac{\partial v_{y}}{\partial t}+\frac{1}{2} \rho_{w} U_{c}^{2} D_{o} C_{L}
\end{array}\right.
$$

In this paper, the van der Pol nonlinear vibration equation was used to describe the shedding characteristics of fluid vortex. According to the discussion on the coupling term of the wake vibrator by [5], when the coupling term is acceleration, the calculation results are more consistent with the experimental results. Therefore, here, the governing equation of the wake oscillator model based on acceleration coupling 
is used and shown as follows:

$$
\left\{\begin{array}{l}
\ddot{q}_{x}+\varepsilon_{x} \omega_{s}^{\prime}\left(q_{x}^{2}-1\right) \dot{q}_{x}+\left(2 \omega_{s}^{\prime}\right)^{2} q_{x}=\frac{A_{x}}{D_{i}} \ddot{v}_{x} \\
\ddot{q}_{y}+\varepsilon_{y} \omega_{s}^{\prime}\left(q_{y}^{2}-1\right) \dot{q}_{y}+\left(\omega_{s}^{\prime}\right)^{2} q_{y}=\frac{A_{y}}{D_{i}} \ddot{v}_{y}
\end{array}\right.
$$

where $q_{x}$ and $q_{y}$ are the dimensionless wake oscillator variables in IL and CF directions respectively; $\omega_{s}^{\prime}\left(=2 \pi S_{t}\left|U_{c}-\dot{v}_{x}\right| / D_{o}\right)$ is the vortex shedding frequency( $S_{t}$ is Strouhal number); and $\varepsilon_{x}, \varepsilon_{y}, A_{x}, A_{y}$ are dimensionless parameters determined by experiment.

The fluctuating drag force coefficient $C_{D}$ and the fluctuating lift force coefficient $C_{L}$ are expressed by the dimensionless wake oscillator variables $q_{x}$ and $q_{y}$, respectively, namely

$$
C_{D}=C_{d}^{\prime} \frac{q_{x}}{2}, \quad C_{L}=C_{l}^{\prime} \frac{q_{y}}{2}
$$

Substituting Eq. (24) into Eq. (22), the final form of external flow force acting on the riser can be obtained as

$$
\left\{\begin{array}{l}
F_{D}(z, t)=\frac{1}{2} \rho_{w} U_{c}^{2} D_{o} \bar{C}_{d}-\rho_{w} U_{c} D_{o} \bar{C}_{d} \frac{\partial v_{x}}{\partial t}+\frac{1}{2} \rho_{w} U_{c}^{2} D_{o} C_{d}{ }^{\prime} \frac{q_{x}}{2} \\
F_{L}(z, t)=-\frac{1}{2} \rho_{w} U_{c} D_{o} \bar{C}_{d} \frac{\partial v_{y}}{\partial t}+\frac{1}{2} \rho_{w} U_{c}^{2} D_{o} C_{l}{ }^{\prime} \frac{q_{y}}{2}
\end{array}\right.
$$

In this study, where $A_{x}=48, A_{y}=12, \varepsilon_{x}=1.2, \varepsilon_{y}=0.3, C_{d}^{\prime}=0.3, C_{l}^{\prime}=0.4$, according to [5], and [48]. (2) Nonlinear contact/collision load between the riser-test pipe (load boundary condition)

A method for calculating the riser-test pipe contact/collision load has been established according to the elastoplastic mechanics theory [47]. The deformation structure is illustrated in Fig. 4, after the contactimpact, the point $A_{2}$ on the test pipe deforms toward point $A_{1}$ on the riser. The geometric relation can be expressed as follows:

$$
R_{i}^{2}=\left(R_{i}-z_{i}\right)^{2}+r^{2} \quad \forall i=1,2
$$

where $R_{1}$ and $R_{2}$ denote the radius of riser and test pipe, respectively $(\mathrm{m}), z_{1}$ and $z_{2}$ are the radial distance from the contact point of pipe to the inner wall of riser, respectively (m), and $r$ is the horizontal distance from the contact point to the test pipe axis (m).

The deformation of riser and test pipe is defined, such that their relative deformation is $\delta(\mathrm{m})$, while the generated contact zone is of width $2 b(\mathrm{~m})$. After contact, the radial displacements of the riser and test pipe are $\omega_{1}$ and $\omega_{2}(\mathrm{~m})$, respectively. From the geometric relation, the relative deformation can be obtained as follows:

$$
\delta=\left(\omega_{2}+\omega_{1}\right)+\left(z_{2}-z_{1}\right)=\left(\omega_{2}+\omega_{1}\right)+\left(\frac{R_{1}-R_{2}}{2 R_{1} R_{2}}\right) r^{2}
$$




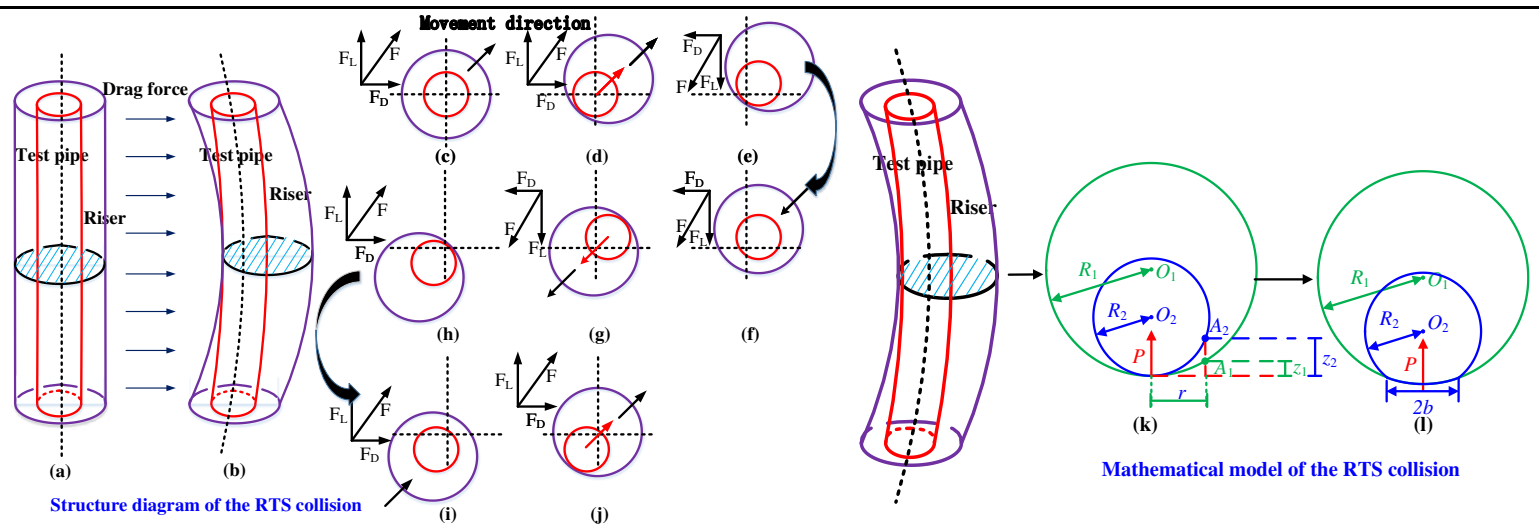

Fig. 4. Contact deformation between the riser and test pipe

If the contact zone width is much smaller than the pipe radius, each pipe can be approximated as an elastic half-plane. According to reference [47], the calculation equations for $\omega_{1}$ and $\omega_{2}$ can be obtained as follows:

$$
\left\{\begin{array}{l}
\omega_{1}=-\frac{2\left(1-\mu_{1}^{2}\right)}{\pi E_{1}}\left[\int_{-b}^{b} q(x) \ln |r-x| \mathrm{d} x-\left(\frac{1}{2\left(1-\mu_{1}\right)}-\ln R_{1}\right) F\right] \\
\omega_{2}=-\frac{2\left(1-\mu_{2}^{2}\right)}{\pi E_{2}}\left[\int_{-b}^{b} q(x) \ln |r-x| \mathrm{d} x-\left(\frac{1}{2\left(1-\mu_{2}\right)}-\ln R_{2}\right) F\right.
\end{array}\right.
$$

where $F$ is contact load of riser-test pipe (N), $E_{1}$ and $E_{2}$ are the elastic modulus of the riser and test pipe material, respectively ( $\mathrm{Pa}), \mu_{1}$ and $\mu_{2}$ represent the Poisson's ratio of riser and test pipe material, respectively, while $q(x)$ is uniform load distribution for riser and test pipe $(\mathrm{N})$, and which is assumed to be proportional to the longitudinal coordinates of the semicircular arc with diameter $2 b$, as follows:

$$
q(x)=\frac{2 F}{\pi b^{2}} \sqrt{b^{2}-x^{2}}
$$

Substituting $E_{1}=E_{2}=E, \mu_{1}=\mu_{2}=0.3$, Eqs. (28), (29) into Eq. (27), the relation between the risertest pipe contact-impact load and deformation can be obtained after simplification, whose detailed derivation is demonstrated in our recent work [39].

$$
\left\{\begin{array}{l}
\delta=1.82 \frac{F}{E}\left(1-\ln \left(1.522 \sqrt{\frac{F}{\pi} \frac{R_{1} R_{2}}{R_{2}-R_{1}}}\right)\right) \\
f_{F}=\xi F
\end{array}\right.
$$

where $\xi$ is the friction coefficient between the riser and test pipe, which can determined using the method proposed by Wen et al. [49] or by performing a wear test. In our present study, a wear test revealed that the friction coefficient is 0.243 for the gas wells in the South China Sea [50].

(3) High-speed fluid impact load in test pipe (load boundary condition)

In the testing condition, when the high-speed fluid in the test pipe passes through regions with changing well inclination angles or deformation area, it generates an impact load on the test pipe (as shown in Fig. 5), resulting in longitudinal and lateral vibrations of the test pipe. According to fluid mechanics [51], 
the high-speed fluid impact load in the test pipe can be calculated using the following equations:

$$
\left\{\begin{array}{l}
f_{x}(x, t)=-\rho_{i} A_{i} V^{2} \sin \left[\alpha_{2}(t)-\alpha_{1}(t)\right] \cos \left[\varphi_{2}(t)-\varphi_{1}(t)\right] \\
f_{y}(x, t)=-\rho_{i} A_{i} V^{2} \sin \left[\alpha_{2}(t)-\alpha_{1}(t)\right] \sin \left[\varphi_{2}(t)-\varphi_{1}(t)\right] \\
f_{z}(x, t)=-\rho_{i} A_{i} V^{2} \cos \left[\alpha_{2}(t)-\alpha_{1}(t)\right]
\end{array}\right.
$$

where $\rho_{i}$ is the density of gas in the test pipe $\left(\mathrm{kg} / \mathrm{m}^{3}\right), A_{i}$ is the cross-sectional area of the wellbore $\left(\mathrm{m}^{2}\right)$; $\alpha_{1}(t), \alpha_{2}(t), \varphi_{1}(t)$ and $\varphi_{2}(t)$ are respectively the deflection angles of the upper and lower microsegments of the test pipe in $x$ - and $y$-directions ( $\mathrm{rad})$, and which are determined by the inclination angle and the deformation of the test pipe.

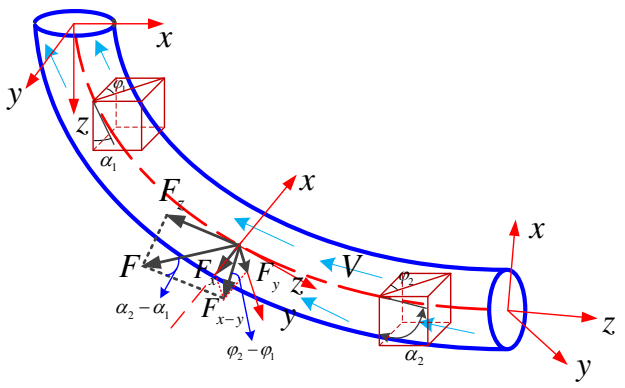

Fig. 5. Schematic of the impact load by high-speed gas

Meanwhile, the inclination angle and azimuth at any depth can be determined through the cubic spline interpolation method [52], which can be expressed as follows in the interval $\left[s_{k-1}, s_{k}\right](k=1,2, \cdots, N)$ :

$$
\left\{\begin{array}{l}
\alpha(s)=\frac{M_{k-1}\left(s_{k}-s\right)^{3}}{6 L_{k}}+\frac{M_{k}\left(s-s_{k-1}\right)^{3}}{6 L_{k}}+C_{k}\left(s-s_{k-1}\right)+C_{k-1}\left(s_{k}-s\right) \\
\varphi(s)=\frac{m_{k-1}\left(s_{k}-s\right)^{3}}{6 L_{k}}+\frac{m_{k}\left(s-s_{k-1}\right)^{3}}{6 L_{k}}+c_{k}\left(s-s_{k-1}\right)+c_{k-1}\left(s_{k}-s\right)
\end{array}\right.
$$

where $C_{j}=\frac{\alpha_{j}}{L_{k}}-\frac{M_{j} L_{k}}{6}, j=k, k-1, \quad c_{j}=\frac{\varphi_{j}}{L_{k}}-\frac{m_{j} L_{k}}{6}, j=k, k-1, \quad M_{j}=\alpha_{j}^{\prime \prime}, j=k, k-1, \quad m_{j}=\varphi_{j}^{\prime \prime}, j=k, k-1, k$ is the order number of measuring point, $L_{k}=s_{k}-s_{k-1}$ is the length of measuring section (m), $s$ is the well depth at the measuring point (m), and $N$ is the total measuring point.

(4) Displacement boundary condition

The different working conditions and the different riser hang-off conditions are mainly different in the upper and lower boundary conditions. In the testing condition, as shown in Fig. 2, the upper end of the riser is connected to the platform with flexible joint (the rotational stiffness of the upper flexible joint is $K_{\mathrm{U}}$ $(\mathrm{N} \cdot \mathrm{m} / \mathrm{deg})$, which can be determined in Mao's recent work [53] and the RTS can move with the movement of the platform. In this study, the heave motion of the platform is mainly considered and the influence of horizontal motion is ignored. That is to say, the horizontal displacement of the platform is 0 . The lower end of the RTS is connected to the BOP and the rotational stiffness is $K_{\mathrm{L}}(\mathrm{N} \cdot \mathrm{m} / \mathrm{deg})$, which also can be determined in Mao's recent work. Therefore, the displacement boundary and initial conditions of the RTS 
under the normal drilling condition can be expressed as:

For the riser:

$$
\left\{\begin{array}{l}
v_{x}(0, t)=0, v_{y}(0, t)=0, v_{z}(0, t)=u_{\text {boat }}(t), v_{x}(L, t)=0, v_{y}(L, t)=0, v_{z}(L, t)=0 \\
\left.E I_{v} v_{x}^{\prime \prime}\right|_{(0, t)}=\left.K_{\mathrm{U}} v_{x}^{\prime}\right|_{(0, t)},\left.E I_{v} v_{y}^{\prime \prime}\right|_{(0, t)}=\left.K_{\mathrm{U}} v_{y}^{\prime}\right|_{(0, t)},\left.E I_{v} v_{x}^{\prime \prime}\right|_{(L, t)}=\left.K_{\mathrm{L}} v_{x}^{\prime}\right|_{(L, t)},\left.E I_{v} v_{y}^{\prime \prime}\right|_{(L, t)}=\left.K_{\mathrm{L}} v_{y}^{\prime}\right|_{(L, t)} \\
v_{x}(z, 0)=0, v_{y}(z, 0)=0, v_{z}(z, 0)=0,\left.v_{x}^{\prime}\right|_{(z, 0)}=0,\left.v_{y}^{\prime}\right|_{(z, 0)}=0,\left.E I v_{x}^{\prime \prime}\right|_{(z, 0)}=0,\left.E I v_{y}^{\prime \prime}\right|_{(z, 0)}=0
\end{array}\right.
$$

For the test pipe:

$$
\left\{\begin{array}{l}
S_{x}(0, t)=0, S_{y}(0, t)=0, S_{z}(0, t)=u_{\text {boat }}(t), S_{x}(L, t)=0, S_{y}(L, t)=0, S_{z}(L, t)=0 \\
\left.E I_{s} S_{x}^{\prime \prime}\right|_{(0, t)}=\left.K_{\mathrm{U}} S_{x}^{\prime}\right|_{(0, t)},\left.E I_{s} S_{y}^{\prime \prime}\right|_{(0, t)}=\left.K_{\mathrm{U}} S_{y}^{\prime}\right|_{(0, t)},\left.E I_{s} S_{x}^{\prime \prime}\right|_{(L, t)}=\left.K_{\mathrm{L}} S_{x}^{\prime}\right|_{(L, t)},\left.E I_{s} S_{y}^{\prime \prime}\right|_{(L, t)}=\left.K_{\mathrm{L}} S_{y}^{\prime}\right|_{(L, t)} \\
S_{x}(z, 0)=0, S_{y}(z, 0)=0, S_{z}(z, 0)=0,\left.E I S_{x}^{\prime}\right|_{(z, 0)}=0,\left.E I S_{y}^{\prime}\right|_{(z, 0)}=0,\left.E I S_{x}^{\prime \prime}\right|_{(z, 0)}=0,\left.E I S_{y}^{\prime \prime}\right|_{(z, 0)}=0
\end{array}\right.
$$

where $u_{\text {boat }}(t)$ is the heave displacement of the platform (m).

(5) Heave motion equation of platform

In the deep water test condition, it is necessary to establish the heave motion model of the platform under the action of random waves to determine the upper boundary displacement of the RTS. According to Shen's work [54], the heave motion equation of platform can be expressed as follows:

$$
m_{\mathrm{p}} \ddot{u}_{\text {boat }}+B_{1} \dot{u}_{\text {boat }}+B_{2} \dot{u}_{\text {boat }}\left|\dot{u}_{\text {boat }}\right|+\rho_{w} g A_{w}\left(u_{\text {boat }}-\eta(t)\right)=\bar{F}_{z}
$$

where $m_{\mathrm{p}}$ is the mass of platform $(\mathrm{m}), B_{1}$ and $B_{2}$ are heave radiation and heave viscous damping, $A_{w}$ is the area of the platform at sea level $\left(\mathrm{m}^{2}\right), \eta(t)$ and $\bar{F}_{z}$ are the surface displacement of random wave (m) and random heave wave exciting force on platform $(\mathrm{N})$.

In this study, using Longuet-Higgins model [55] to simulate random wave surface displacement, which can be expressed as follows:

$$
\eta(t)=\sum_{i=1}^{M} a_{i} \cos \left[\hat{\omega}_{i} t+\varepsilon_{i}\right]
$$

where $\hat{\omega}_{i}\left(=\left(\omega_{i-1}-\omega_{i}\right) / 2\right)$ is circular frequency of the $i$ th harmonic $(\mathrm{Hz}), \varepsilon_{i}$ is the initial phase of the $i$ th harmonic component (rad), taking the random number in the range $(0,2 \pi) ; M$ is the interval number of partition; $a_{i}(=\sqrt{2 S(\omega) \Delta \omega})$ is amplitude of the $i$ th harmonic component $(\mathrm{m}) ; \Delta \omega\left(=\left(\omega_{H}-\omega_{L}\right) / N\right)$ is the frequency step, $S(\omega)$ is the random wave spectrum, which was described using JONSWAP spectrum in this study. The expression can be obtained in the Shen's work [54]:

$$
\left\{\begin{array}{l}
S(\omega)=\frac{0.06238(1.094-0.01915 \ln \gamma)}{0.23+0.0336 \gamma-0.185(1.9+\gamma)^{-1}} H_{1 / 3}^{2} T_{P}^{-4} f^{-5} \exp \left[-\frac{5}{4}\left(T_{P} f\right)^{-4}\right] \gamma^{\exp \left[-\left(f / f_{p}-1\right)^{2} / 2 \sigma^{2}\right]} \\
T_{p}=\frac{T_{1 / 3}}{1-0.132(\gamma+0.2)^{-0.559}}, \sigma=\left\{\begin{array}{l}
0.07, f \leq f_{p} \\
0.09, f>f_{p}
\end{array}\right.
\end{array}\right.
$$


where $f$ is the frequency $(\mathrm{Hz})$, and $\omega$ is the circular frequency $(\mathrm{Hz}), \omega=2 \pi f ; H_{1 / 3}$ and $T_{1 / 3}$ is the significant wave height (m) and significant period (s) of the wave, $f_{p}$ and $T_{p}$ are peak frequency $(\mathrm{Hz})$ and peak period (s) of the wave, $\gamma$ is peak parameters, which is 3.3 in this study, $\sigma$ is the peak shape coefficient.

Also, according to Shen's work, the random heave wave exciting force on platform includes two parts, one is the exciting force of the random wave on the platform body $\left(F_{s}(t)\right)$, the other is the exciting force of the random wave on the heave plate $\left(F_{p}(t)\right)$, which can be expressed as follows:

$$
\bar{F}_{z}=F_{s}(t)+F_{p}(t)=2 \rho_{w} g \pi R^{2}\left[1-\frac{1}{2} \sin (k R)\right]\left(\frac{J_{1}(k R)}{k R}\right) e^{-k d} \eta(t)+0.597 \frac{\rho_{w} g}{4} \omega^{2} e^{k z_{\text {plate }}} B_{\text {plate }}^{3} \eta(t)
$$

where $R$ is platform radius (m), $k$ is wave number, $J_{1}(\cdot)$ is the first order Bessel function of first kind, $d$ is the draft of platform (m), $z_{\text {plate }}$ is the depth of heave plate $(\mathrm{m}), B_{\text {plate }}$ is the width of heave plate $(\mathrm{m})$.

\subsection{Solution scheme}

This study used the linear Lagrange and cubic Hermitian functions to express the longitudinal/transverse displacements of riser and test pipe; the finite element discrete forms can be expressed as follows:

$$
\left\{\begin{array}{lll}
v_{x}=\boldsymbol{\varphi}_{x}^{\mathbf{T}} \mathbf{d} & v_{y}=\boldsymbol{\varphi}_{y}^{\mathbf{T}} \mathbf{d} & v_{z}=\boldsymbol{\varphi}_{z}^{\mathbf{T}} \mathbf{d} \\
S_{x}=\boldsymbol{\varphi}_{x}^{\mathbf{T}} \overline{\mathbf{d}} & S_{y}=\boldsymbol{\varphi}_{y}^{\mathbf{T}} \overline{\mathbf{d}} & S_{z}=\boldsymbol{\varphi}_{z}^{\mathbf{T}} \overline{\mathbf{d}}
\end{array}\right.
$$

where, $\mathbf{d}$ and $\overline{\mathbf{d}}$ are the displacement vectors of riser and test pipe unit, respectively; $\boldsymbol{\varphi}_{x}, \boldsymbol{\varphi}_{y}$ and $\boldsymbol{\varphi}_{z}$ are respectively represent the vibration shape function of riser and test pipe unit, which are expressed as:

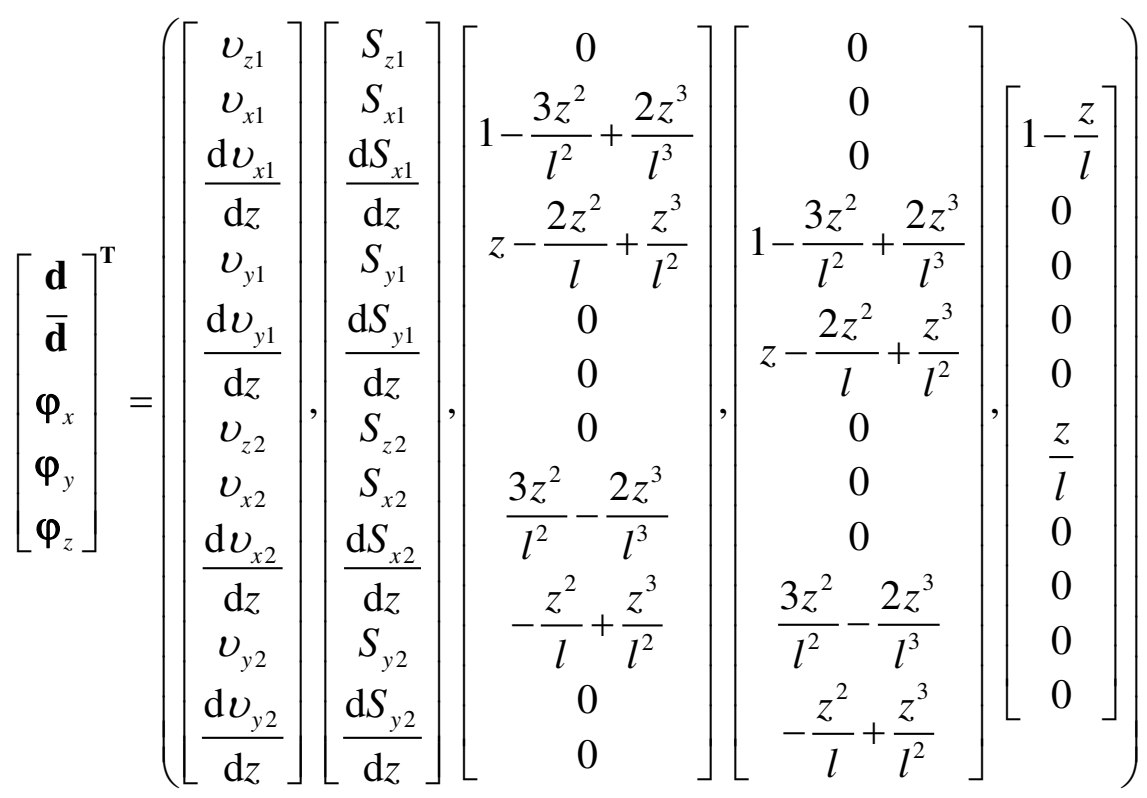

By substituting the displacement obtained from Eqs. (39) and (40) into the energy functional of the RTS, the standard forms of the strain energy function $U$, kinetic energy function $T$ and energy function with external force $W$ expressed by the node displacement vectors can be obtained. After assembling the 
structural elements, the discrete dynamic equation of the system can be obtained according to the variational principle:

$$
\mathbf{M}(t) \mathbf{C}(t) \boldsymbol{\$}+\mathbf{K}(t) \mathbf{D}=\mathbf{F}(t)
$$

where D represents the matrix of overall displacement, which is given by Eq. (39), and $\mathbf{F}(t)$ represents the load column vector of the structure, which accounts for the impact force of gas on the test pipe as well as the contact/friction force of the tubing-casing, ocean environment load and the expressions of them are shown in Section 2.2. $\mathbf{K}(t), \mathbf{M}(t)$, and $\mathbf{C}(t)$ represent the matrices of the overall stiffness, mass, and damping, respectively.

Because there are too many nonlinear factors considered in the 3D nonlinear vibration model, the calculation accuracy of model will be decreased and the model will be difficult to converge if only using Newmark- $\beta$ method for gradual integration. Therefore, in this study, the incremental Newmark- $\beta$ method and Newton-Raphson method are used to solve the discrete Eq. (41) simultaneously, and the specific derivation process can be seen in our previous work [56]. The wake vibrator (shown in Eq. (23)) was solved by a fourth-order Runge-Kutta technique [22]. The coupling iteration between the Runge-Kutta technique and incremental Newmark- $\beta$ method was performed to determine the dynamic response of the RTS. The solution flow of the nonlinear dynamic model is shown in Fig. 6 and its FORTRAN calculation code was developed.

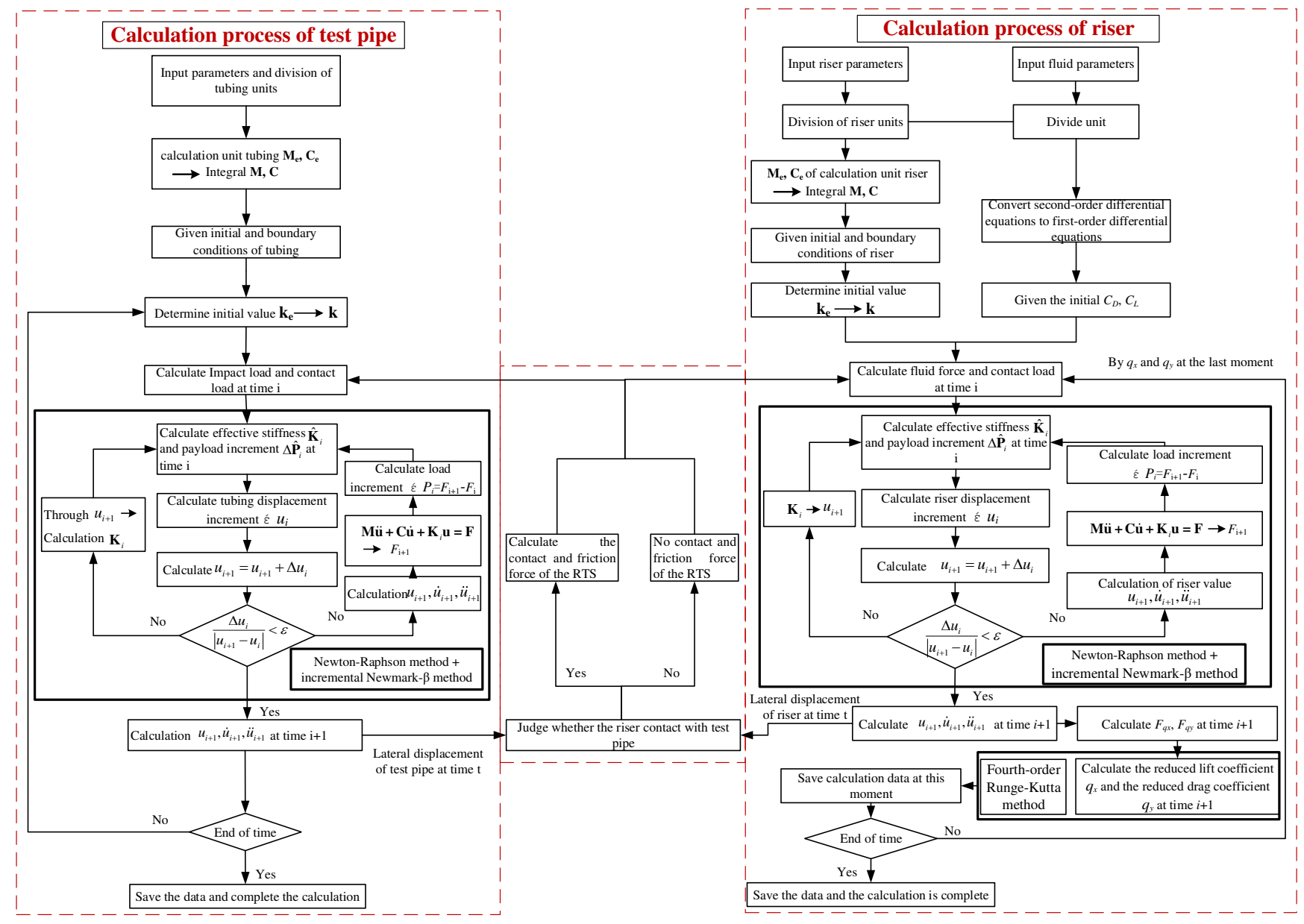

Fig. 6. Flow chart of solving nonlinear vibration model of the RTS 


\subsection{Experimental verification}

(1) Simulated experimental parameters

Because the vibration data of deep-water RTS cannot be accurately measured onsite, a simulation experiment was performed to validate the nonlinear vibration model in this study. Three criteria should be satisfied for the similarity experiment of RTS vibration: geometric similarity, motion similarity, and dynamic similarity $[57,58]$. Based on field parameters of BY-M gas well in deep-water block of South China Sea and simulation experiment area conditions, the basic sizes of the riser and test pipe (inner diameter, outer diameter, tube length, etc.) in the simulation experiment were determined using geometric similarity. Because the size difference between the length and radial directions was considerable, the uniform scale ratio was not adopted. The similarity ratios in the radial and length directions were set as 7.14 and 193.2, respectively. According to our recent work [39], the material density and elastic modulus of the experimental pipe string and the actual pipe string should satisfy the following:

$$
\frac{E_{\mathrm{p}} / E_{\mathrm{m}}}{\rho_{\mathrm{p}} / \rho_{\mathrm{m}}}=\lambda=7.14
$$

where $\rho_{\mathrm{p}}$ and $E_{\mathrm{p}}$ represent the density $\left(\mathrm{kg} / \mathrm{m}^{3}\right)$ and elastic modulus $(\mathrm{Pa})$ of the actual RTS, respectively; $\rho_{\mathrm{m}}$ and $E_{\mathrm{m}}$ represent the density $\left(\mathrm{kg} / \mathrm{m}^{3}\right)$ and elastic modulus $(\mathrm{Pa})$ of the RTS in the simulation experiment, respectively; and $\lambda$ represents the principal similarity ratio (radial similarity ratio). Thus, by substituting the density $\left(\rho_{\mathrm{p}}=7850 \mathrm{~kg} / \mathrm{m}^{3}\right)$ and elastic modulus $\left(E_{\mathrm{p}}=206 \mathrm{GPa}\right)$ of the actual RTS into Eq. (42), we obtain the following:

$$
\frac{E_{\mathrm{m}}}{\rho_{\mathrm{m}}}=\frac{1}{7.14} \frac{E_{\mathrm{p}}}{\rho_{\mathrm{p}}} \approx 3.68 \times 10^{-3} \mathrm{GPa} \cdot\left(\mathrm{kg} / \mathrm{m}^{3}\right)^{-1}
$$

According to the elastic modulus/density ratio, the PVC tube was selected to satisfy the requirement by referring to the metal materials manual [59]. Its elastic modulus and density were $E_{\mathrm{m}}=3.92 \mathrm{GPa}$ and $\rho_{\mathrm{m}}=1150 \mathrm{~kg} / \mathrm{m}^{3}$, respectively.

According to the dynamic similarity, the fluid velocity of the simulated experiment is consistent with the actual fluid velocity onsite. Thus, we can obtain the experimental gas flow, which is $239.95 \mathrm{~m}^{3} / \mathrm{d}$, and the rated pressure, motor power, and maximum flow of the air compressor are 1.25 MPa, $2200 \mathrm{~W}$, and 302.4 $\mathrm{m}^{3} / \mathrm{d}$, respectively, which can satisfy the requirements of the simulation experiment (as shown in Fig. 8(e)). To accurately determine the vibration amplitude and frequency of the pipe, strain gauges are used to measure the strain characteristics at different locations of the pipe. All parameters of the final simulation experiment are shown in Table 1.

\begin{tabular}{|c|c|c|c|c|c|c|c|c|c|}
\hline Parameter & Test pipe & Test pipe & Test & Riser & Riser & Gas & Elastic & Densi & External \\
\hline
\end{tabular}

Table 1. Simulated experiment parameters 


\begin{tabular}{cccccccccc}
\hline & $\begin{array}{c}\text { outer } \\
\text { diameter } \\
(\mathrm{m})\end{array}$ & $\begin{array}{c}\text { inner } \\
\text { diameter } \\
(\mathrm{m})\end{array}$ & $\begin{array}{c}\text { pipe } \\
\text { length } \\
(\mathrm{m})\end{array}$ & $\begin{array}{c}\text { outer } \\
\text { diameter } \\
(\mathrm{m})\end{array}$ & $\begin{array}{c}\text { inner } \\
\text { diamet } \\
\mathrm{er}(\mathrm{m})\end{array}$ & $\begin{array}{c}\text { flow } \\
\text { velocit } \\
\mathrm{y}(\mathrm{m} / \mathrm{s})\end{array}$ & $\begin{array}{c}\text { modulus } \\
(\mathrm{GPa})\end{array}$ & $\begin{array}{c}\text { ty } \\
(\mathrm{kg} / \\
\left.\mathrm{m}^{3}\right)\end{array}$ & $\begin{array}{c}\text { velocity } \\
(\mathrm{m} / \mathrm{s})\end{array}$ \\
\hline $\begin{array}{c}\text { Actual } \\
\text { parameter } \\
\text { Test }\end{array}$ & 0.1143 & 0.095 & 579.7 & 0.2445 & 0.2168 & 18.05 & 206.0 & 7850 & 0.5 \\
parameter & 0.016 & 0.014 & 3.0 & 0.034 & 0.030 & 18.05 & 3.92 & 1150 & 0.5 \\
\hline
\end{tabular}

(2) Simulation experimental system

The experimental system is mainly composed of experimental pool, experimental bench, string system, data testing system and gas supply system (as shown in Fig. 7). The size of experimental pool is $30 \mathrm{~m} \times 15 \mathrm{~m} \times 3 \mathrm{~m}$ (as shown in Fig. $8(\mathrm{f})$ ). The experimental bench is mainly composed of experimental steel frame which is welded by H-beam (12.5m long and 3.5m high), poplar roller, fixed pulley, wire rope, track, etc. (as shown in Fig. 8(a)), and its function is to change the movement direction of the wire rope using the fixed pulley, so as to ensure that the movement direction and speed of the top and bottom wire ropes are consistent. Meanwhile, using the wire rope to drag the experimental string can realize the synchronous movement of the string, so as to ensure the force of the simulated uniform current. The experimental string system consists of inner and outer simulated tubes and upper and lower joint simulators, in which the upper end of the string is connected with the slider by a spring with elastic stiffness, and the lower end is connected with the lower slider by a rotatable universal joint (as shown Figs. 8(b), (c) and (d)). The data test system consists of strain gauge, shield wire and data acquisition instrument, in which the measurement accuracy and sampling frequency of the strain gauges are $7.5 \mu \varepsilon$ and $500 \mathrm{~Hz}$, eight measurement points are arranged around the pipe, for a total of 32 points. Nodes 1 and 8 are $0.17 \mathrm{~m}$ from the ends of the pipe, and eight measurement points are evenly arranged along the length direction of the pipe at intervals of $0.38 \mathrm{~m}$. The gas supply and transportation system consists of screw air compressor, high pressure gas storage tank, connecting pipeline, flowmeter, solenoid valve, etc.. The physical diagrams of each part of the experimental system are shown in Fig. 8.

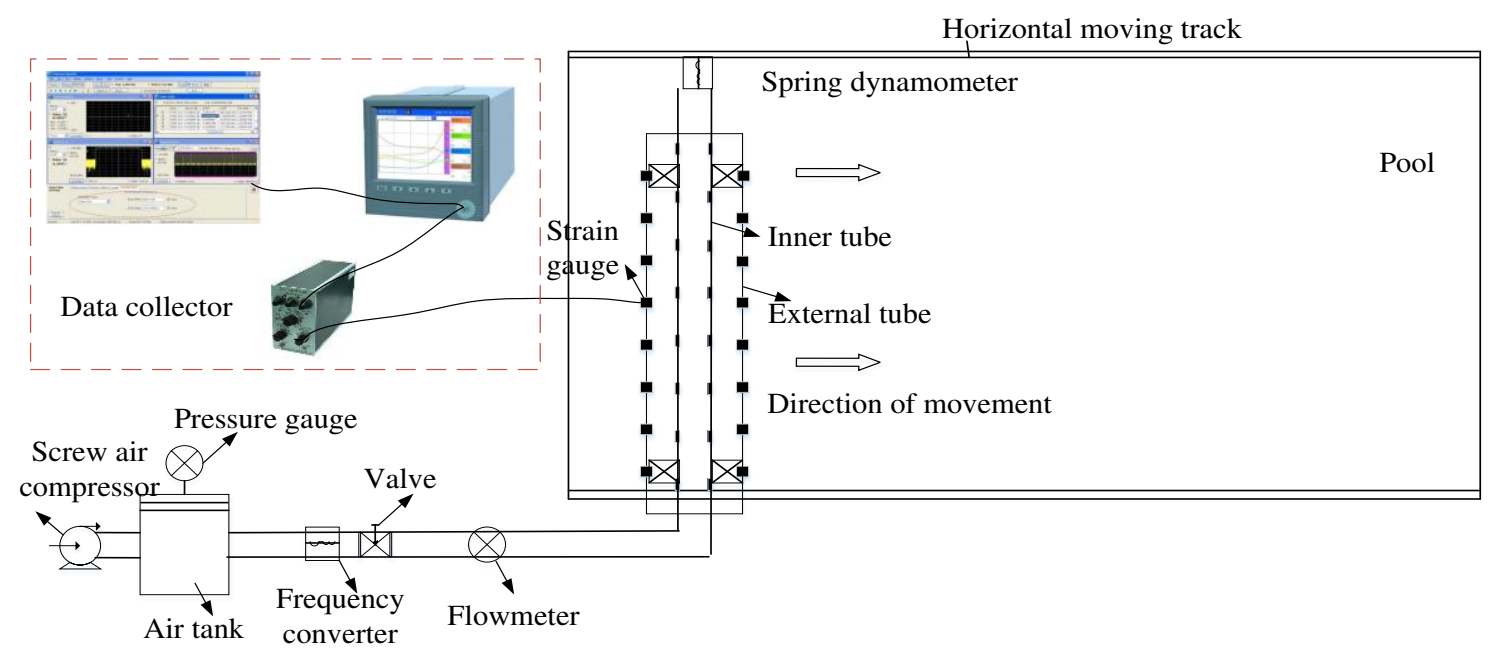

Fig. 7. Experimental system design 


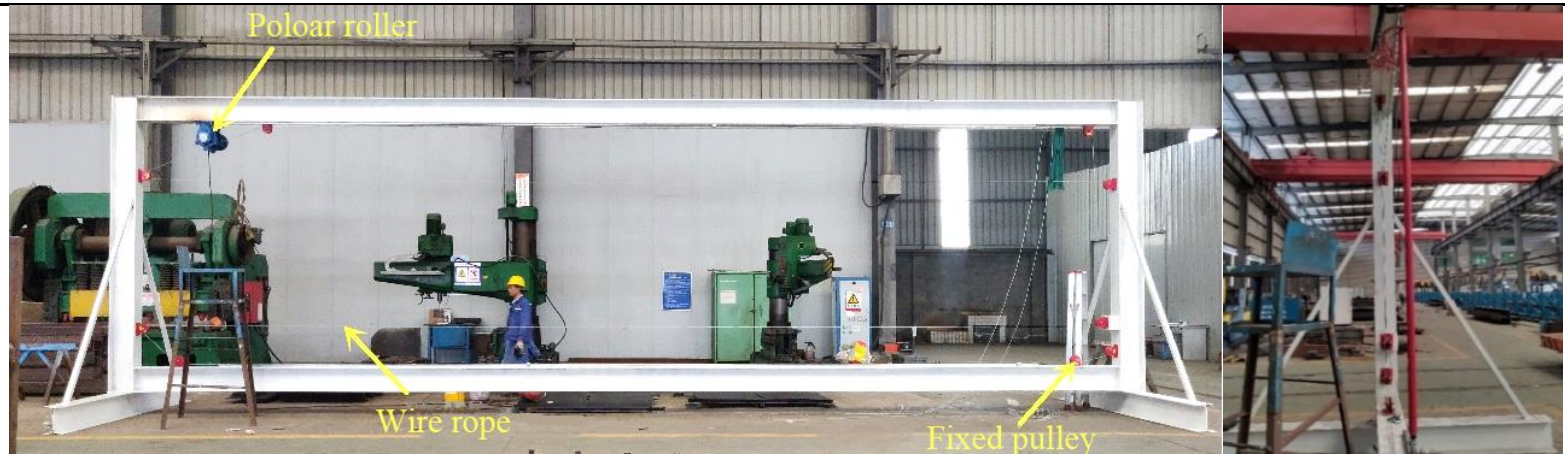

(a) Physical drawing of test bench

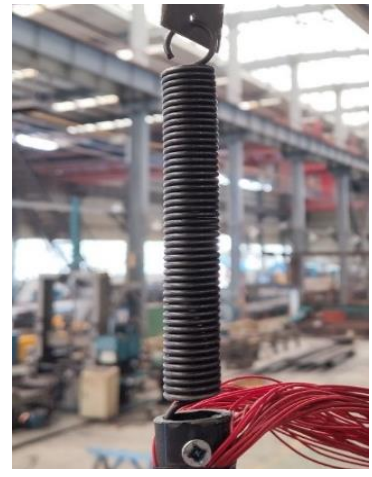

(b) Adjusting spring

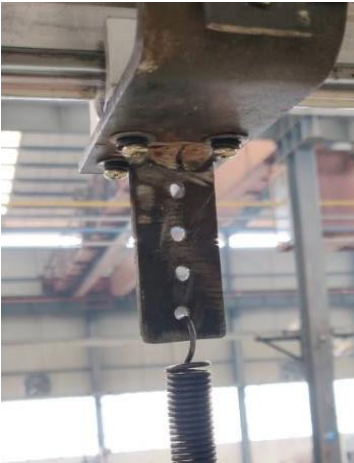

(c) Tension adjustment device

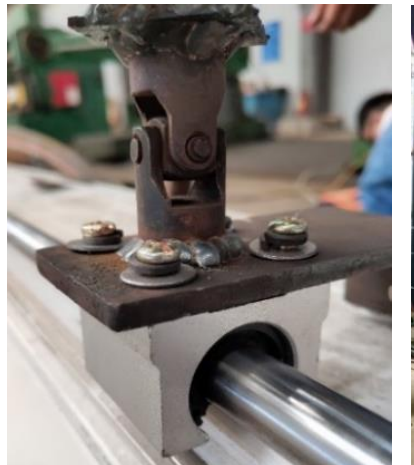

(d) Bottom universal joint

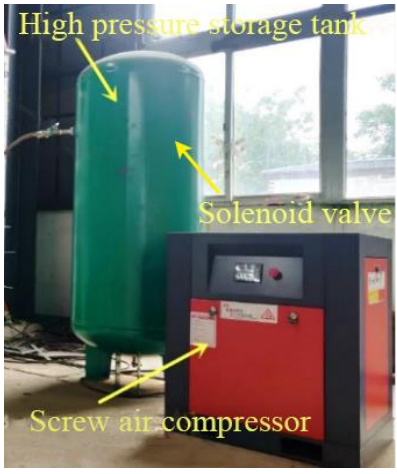

(e) Gas transportation system

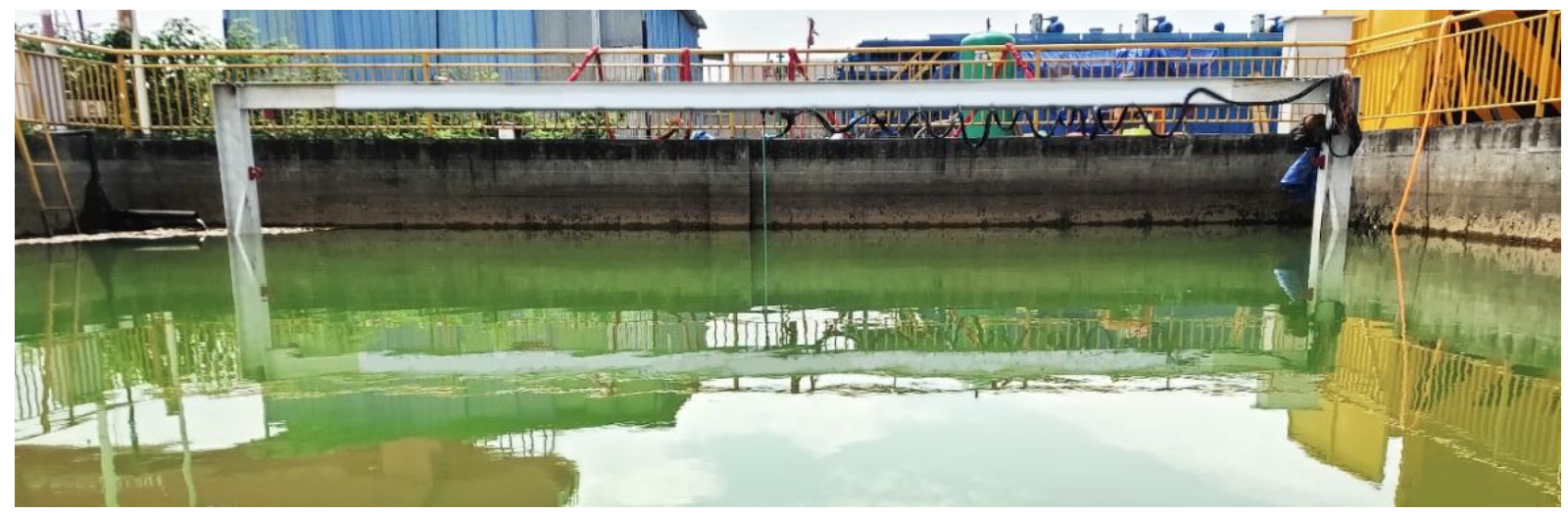

(f) Experimental pool

Fig. 8. Physical diagram of test system

(3) Experimental results

In the experiment, eight measuring points are arranged on the surface of the inner and outer pipe, and four groups of strain gauges are arranged on the section of the same measuring point, which are uniformly distributed $90^{\circ}$ along the circumference of the pipe. The vibration characteristics of the pipe in the flow direction are measured by in-line flow 1 (IL1) and in-line flow 2 (IL2), and that in the vertical flow direction are measured by cross-flow 1 (CF1) and cross-flow 2 (CF2), as shown in Fig. 6b. According to the theoretical model established in the first section, the single riser vibration model [22] and the single flow induced vibration model of the test pipe [39] in our recent researches, the vibration response of the RTS calculated using the same parameters as the test parameters (as shown in Table 1), in which, the riser and test pipe were divided into 300 units. The simulation time was 10s, and the step size was $0.001 \mathrm{~s}$.

Figs. 9 and 10 show the root mean square (RMS) distribution and the amplitude frequency response 
curve (at the measuring point 3) of the riser displacement in IL and CF direction. It can be seen from Fig. 9 that the CF RMS of the riser was significantly greater than the IL RMS. Through the comparison of the experimental data, the calculation results using the proposed model in section 2.1 and the single riser model in our recent work [22], it is found that the calculation results of the proposed model were closer to the Meanwhile, which verified the correctness and effectiveness of the proposed model. It can be discovered in Fig. 10 that, regardless of the experimental results and the theoretical calculation results, the IL vibration frequency of the riser was twice that of the CF vibration frequency, which was consistent with the previous research results $[11,22]$. The amplitude of the riser using the single vibration model was the largest, and the main reason was that there was no restraining effect of the test pipe. By observing the experimental amplitude frequency response of the measurement point 3 , it is found that the vibration was obviously complex, which was mainly affected by the external factors in the test process. Meanwhile, compared with calculation results using the single vibration model, the calculation results in this paper were closer to the experimental results, which verifies the correctness of the RTS vibration model again.

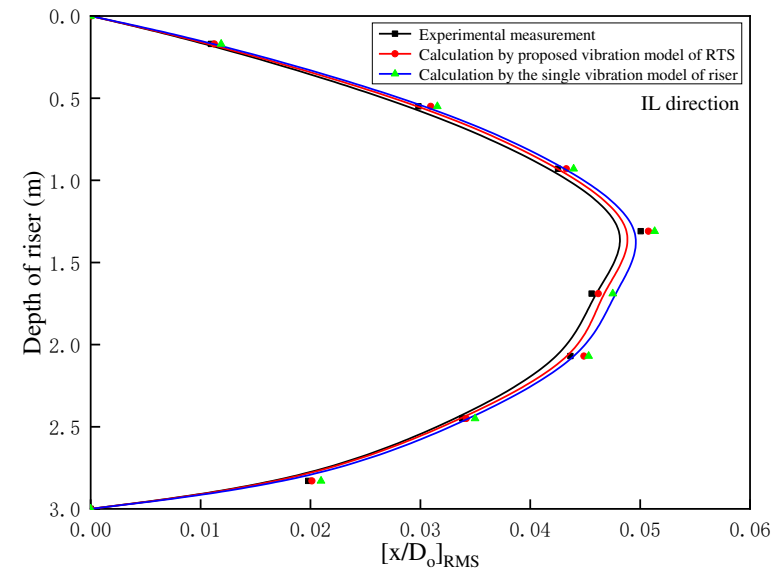

(a) The RMS of riser in IL direction

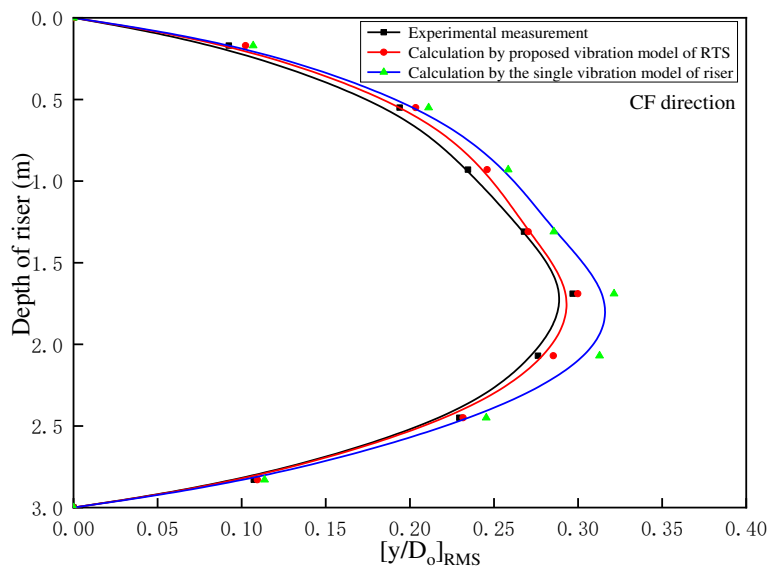

(b) The RMS of riser in CF direction

Fig. 9. The RMS distribution of riser displacement

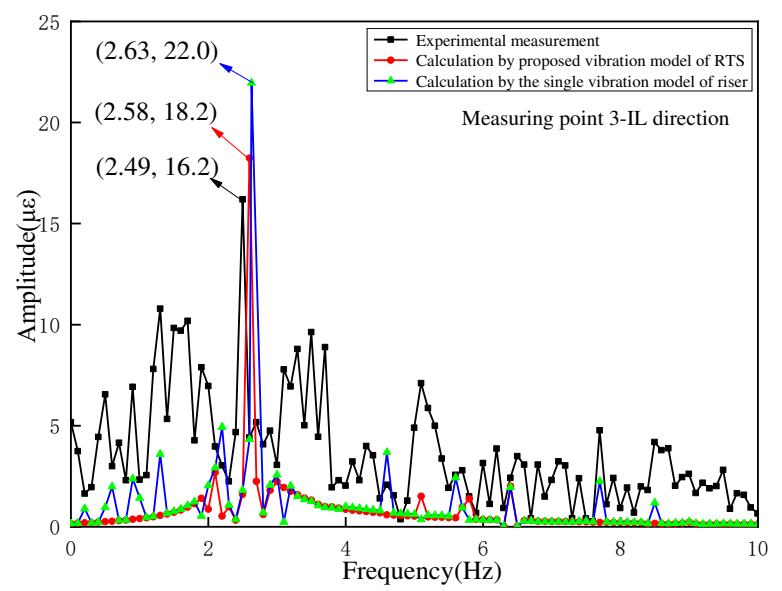

(a) The amplitude frequency response curve of riser in IL direction

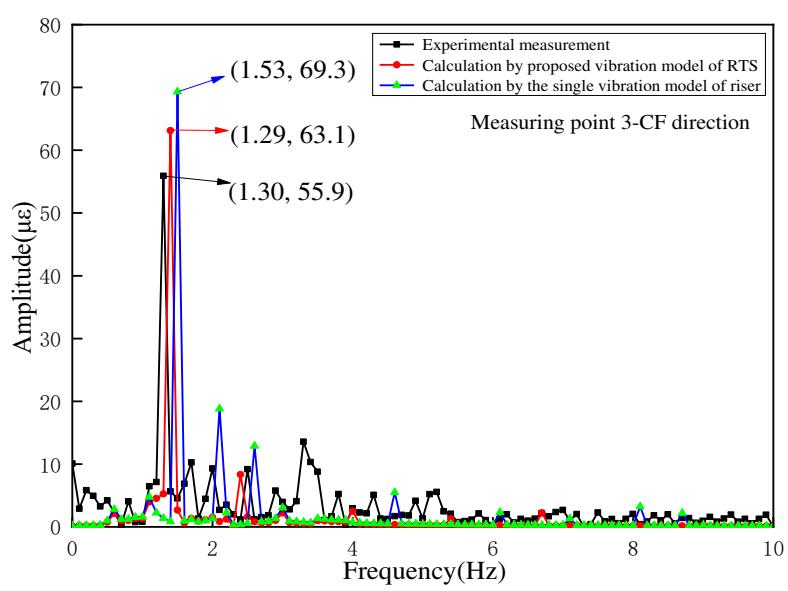

(b) The amplitude frequency response curve of riser in CF direction

Fig. 10. The Amplitude frequency response curve of riser at measuring point 3

Figs. 11 and 12 show the RMS distribution and the amplitude frequency response curve (at the 
measuring point 3) of the test pipe displacement in IL and CF direction. It can be noted in Fig. 11 that when the riser was fixed and only the flow induced vibration (FIV) of the test pipe was considered, the calculated results of test pipe were significantly smaller than the experimental results and the calculation results in this paper, which indicates that the vibration of the test pipe was mainly caused by the riser vibration. Therefore, the nonlinear vibration of the riser cannot be ignored in the vibration analysis of the test pipe, and the calculation results of test pipe obtained using the proposed nonlinear model of RTS can be closer to reality. It can be observed in Fig. 12 that, the main frequency obtained by the single test pipe vibration model was significantly lower than that of the test and the calculation results of proposed model. It shows again that the influence of riser should be considered when analyzing the vibration response of the test pipe, and the effectiveness of the nonlinear model was verified. Comparing the calculation results using proposed model and the experimental results, the main frequency and amplitude obtained by the two methods were very similar, which verified the correctness of the nonlinear model of RTS in this paper, and lays the foundation for the fatigue reliability analysis of test pipe in Section 4.

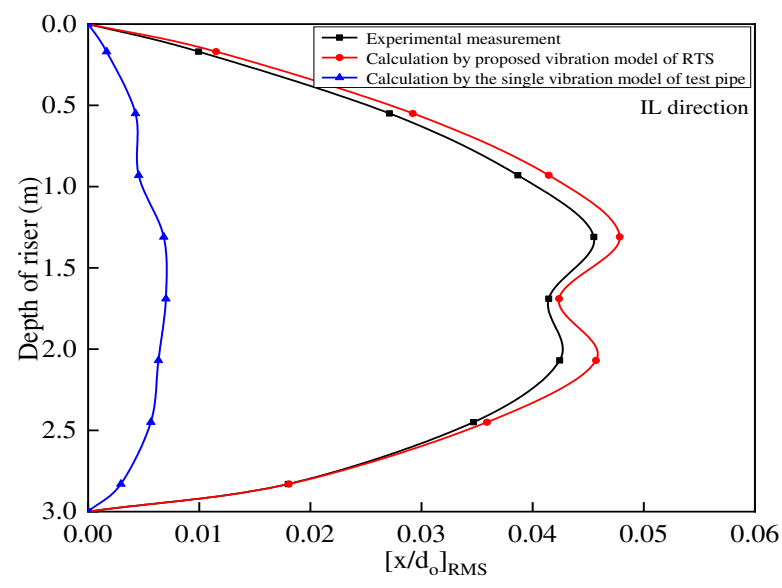

(a) The RMS of riser in IL direction

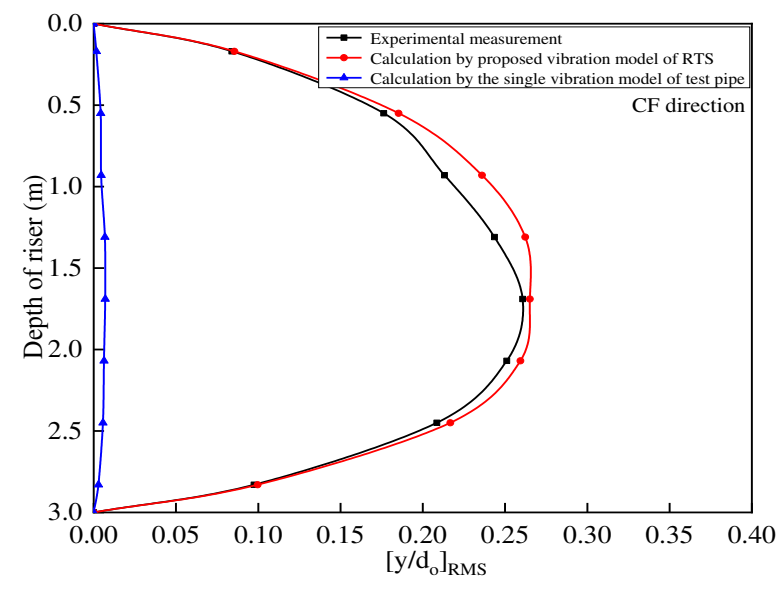

(b) The RMS of riser in CF direction

Fig. 11. The RMS distribution of test pipe displacement

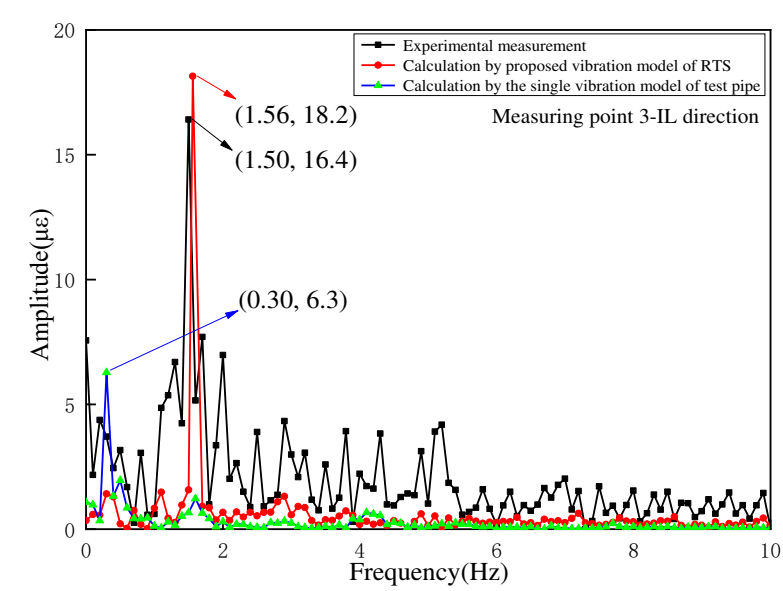

(a) The amplitude frequency response curve of test pipe in IL direction

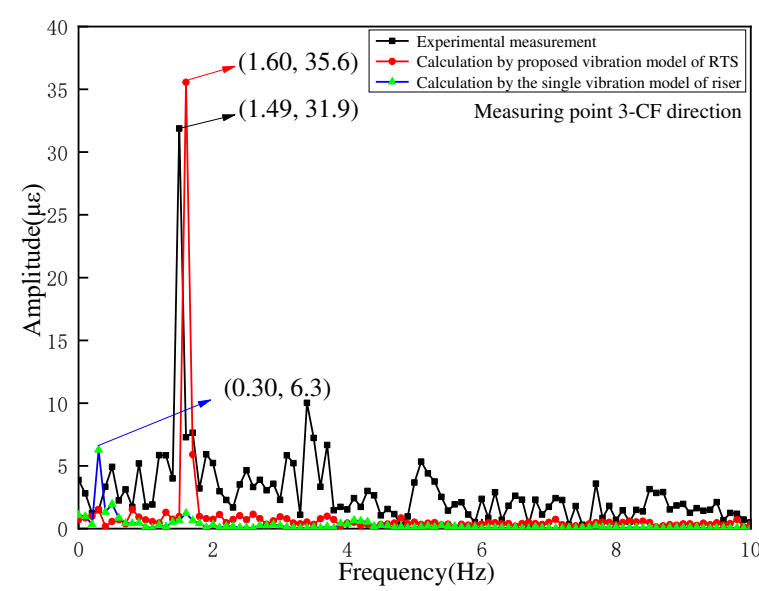

(b) The amplitude frequency response curve of test pipe in $\mathrm{CF}$ direction

Fig. 12. The Amplitude frequency response curve of test pipe at measuring point 3 


\subsection{Fatigue life prediction model}

In this section, the cumulative damage theory (Miner's Law) is used to predict the fatigue life of the test pipe [60]. The principle is that, when fatigue damage occurs under multi-stage constant amplitude alternating stress, the total damage is the sum of the fatigue damage components under all levels of stress cycles, and the steps of fatigue life analysis are show as follows.

The stress amplitude of all stress cycles in the service life of the test pipe $\left(\sigma_{1}, \sigma_{2}, \sigma_{3} \ldots\right)$ can be determined by the 3D nonlinear vibration model in Section 2, and the corresponding number of cycles $\left(n_{1}, n_{2}, n_{3} \ldots\right)$ can be calculated by the rain flow counting method [61]. The number of cycles of each stress amplitude acting alone $\left(N_{1}, N_{2}, N_{3} \ldots\right)$ can be obtained from the S-N curve of the test pipe. So, the damage degree caused by each stress cycle can be obtained $\left(n_{1} / N_{1}, n_{2} / N_{2}, n_{3} / N_{3} \ldots\right)$. The total fatigue damage of the test pipe can be obtained by adding all the stress cycle damage, which can show as following:

$$
D^{\prime}=\frac{n_{1}}{N_{1}}+\frac{n_{2}}{N_{2}}+\frac{n_{3}}{N_{3}}+\cdots=\sum_{i=1} \frac{n_{i}}{N_{i}}
$$

The fatigue life of the test pipe is the reciprocal of the total fatigue damage, which can show as following:

$$
T_{f}=\frac{1}{D^{\prime}}
$$

where, $T_{f}$ is the service life of test pipe and $D^{\prime}$ is the total fatigue damage.

Finally, the flow chart of fatigue life analysis of test pipe is obtained, as shown in Fig. 13. According to the fatigue life analysis method of test pipe, the $\mathrm{S}-\mathrm{N}$ curve of pipe material is necessary for the calculation of the fatigue life. Therefore, the next step is to carry out fatigue experiment to determine the S-N curve of the test pipe.

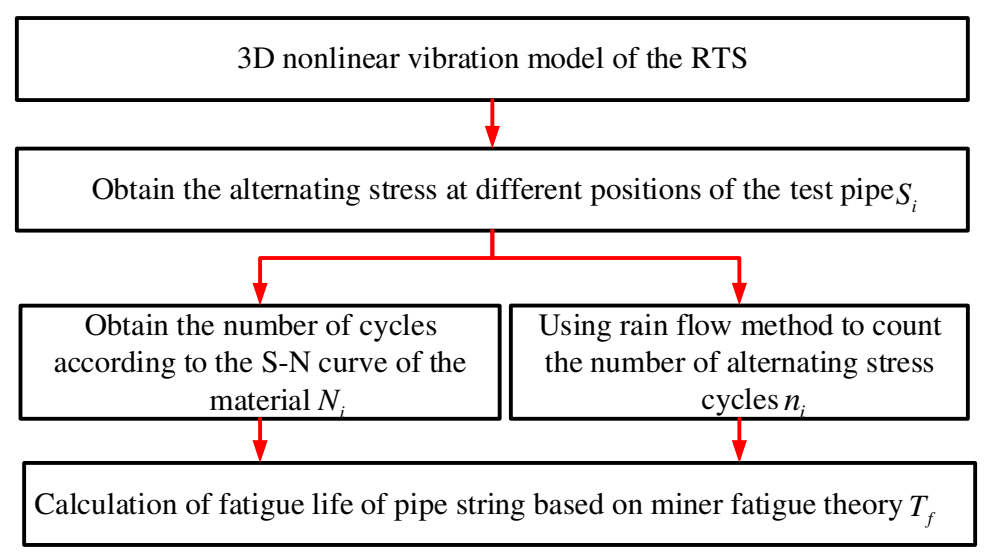

Fig. 13. Flow chart of fatigue life analysis of test pipe

\subsection{Experiment measurement of $S-N$ curve}

(1) Equipment of experiment

PQ-6 pure bending fatigue tester is used for this fatigue experiment (Fig. 14), and the working principle of which is that both ends of the test piece are clamped by clamps, and the test load is determined by the 
weights. So, if the minimum diameter of the specimen is $d_{\min }$, the weight can be calculated by the following formula.

$$
F^{\prime}=\frac{\pi d_{\min }^{3} \sigma}{16 L}-G
$$

where $F^{\prime}$ is weight of loading weight $(\mathrm{N}), L=L_{1}=L_{2}$ are $\operatorname{arm}$ length $(\mathrm{m}), \sigma$ is stress of test piece $(\mathrm{Pa}), G$ is additional weight including weight plate and force bracket $(\mathrm{N})$.

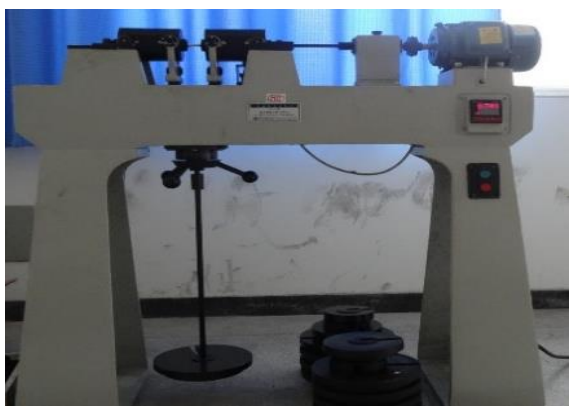

(a)

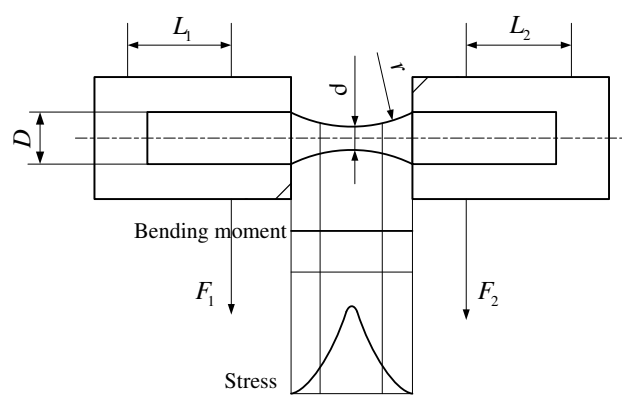

(b)

Fig. 14. PQ-6 pure bending fatigue testing machine (a) and stress analysis diagram of test specimen (b)

(2) Experimental procedure

(1) Minimum diameter of test specimen is measuring.

(2) According to the determined stress level, calculate the weight of the added weight by Eq. (46), and plus weights.

(3) Lift the weight before starting the machine, add the weight quickly and without impact after the running is stable, and set the counter to zero.

(4) If the sample breaks or the number of tests exceeds $10^{7}$, record the number.

(3) Experimental result

The determination of S-N curve is completed in two parts which are limited fatigue life zone and infinite fatigue life zone, as shown in Fig. 15.

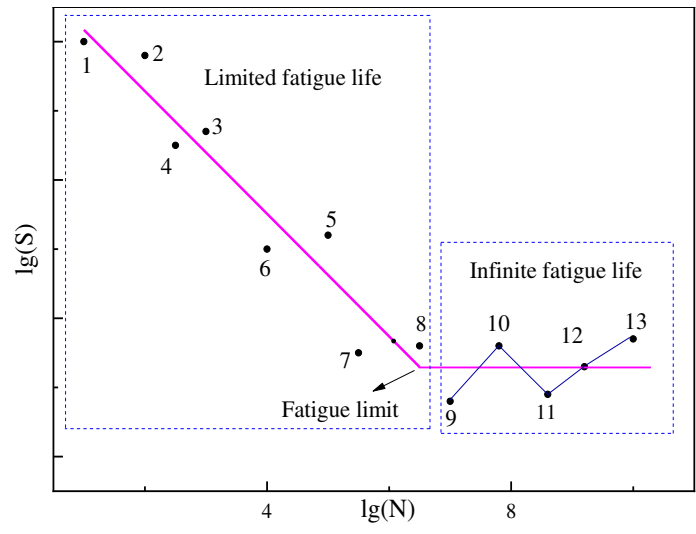

Fig. 15. Schematic diagram of measuring S-N curve

(1) Limited fatigue life zone

Due to the yield limit of pipe material (13Cr-L80) is $665 \mathrm{MPa}$, seven stress amplitudes are preliminarily set as $585 \mathrm{MPa}, 535 \mathrm{MPa}, 485 \mathrm{MPa}, 435 \mathrm{MPa}, 425 \mathrm{MPa}, 415 \mathrm{MPa}$ and $405 \mathrm{MPa}$. Three test specimens were 
measured under each stress amplitude, and the number of cycles were recorded when each test specimen broke. Then, the average number of cycles of three test specimens was taken as the number of cycles corresponding to the stress amplitude, and 21 test specimens were measured in total (Table 2). If the number of cycles of the minimum stress amplitude has not exceeded $10^{7}$, reduce the stress amplitude and continue the test until the number of cycles is greater than $10^{7}$. Therefore, the S-N curve of finite fatigue life zone can be obtained.

Table 2. Test data of limited fatigue life zone

\begin{tabular}{cccc}
\hline $\begin{array}{c}\text { Stress amplitude S } \\
(\mathrm{MPa})\end{array}$ & Cycle times N & $\begin{array}{c}\text { The logarithmic form of the } \\
\text { number of cycles }(\operatorname{lgN})\end{array}$ & $\begin{array}{c}\text { Logarithmic form of stress } \\
(\operatorname{lgS})\end{array}$ \\
\hline 585 & 14611 & 4.16 & 2.77 \\
535 & 42749 & 4.63 & 2.73 \\
485 & 79520 & 4.90 & 2.68 \\
435 & 258661 & 5.41 & 2.63 \\
425 & 289239 & 5.46 & 2.62 \\
415 & 540381 & 5.73 & 2.61 \\
405 & 7914224 & 6.79 & 2.60 \\
\hline
\end{tabular}

(2) Infinite fatigue life zone

The initial stress amplitude is determined by the fatigue limit range obtained from the previous test, and the fatigue limit is measured by the lifting method, and the infinite fatigue life zone is obtained by the fatigue limit. According to the judgment of the first sample, if the first sample fails, the stress value will be reduced. Otherwise, it increases (Table 3). The fatigue limit of 13Cr-L80 is calculated from the data of the effective test specimen by formula (25), and the S-N curve of the infinite fatigue life zone is obtained.

$$
\sigma_{-1}=\frac{1}{9} \times(2 \times 425+4 \times 405+3 \times 385)=402.78 \mathrm{MPa}
$$

Table 3. Test data of infinite fatigue limit zone

\begin{tabular}{cccc}
\hline Number & Stress $(\mathrm{MPa})$ & Cycle times & Result \\
\hline 1 & 385 & 10654984 & Suspension \\
2 & 405 & 10343146 & Suspension \\
3 & 425 & 323889 & Invalid \\
4 & 405 & 10153919 & Suspension \\
5 & 425 & 307439 & Invalid \\
6 & 405 & 3245607 & Invalid \\
7 & 385 & 10438520 & Suspension \\
8 & 405 & 1018500 & Invalid \\
9 & 385 & 10248537 & Suspension \\
\hline
\end{tabular}

\subsection{S-N curve fitting and correction}

After the S-N curve of pipe material is obtained, in order to make the curve more consistent with the actual situation of the field pipe, the S-N curve was corrected from the following four aspects.

(1) Load type correction factor $C_{L}$ 
In the actual working condition, the test pipi is subject to vertical and horizontal coupling, while the test piece is subject to pure bending only. Therefore, it is necessary to correct the S-N curve measured in the test, and the correction factor of load type is 0.7 from Table 4.

Table 4. Load type correction factor $C_{L}[62]$

\begin{tabular}{ccccr}
\hline Loading type & Pure bending load & Axial load (Slightly bent) & Reverse about steel & Reverse about cast iron \\
\hline$C_{L}$ & 0.9 & 0.7 & 0.58 & 0.8 \\
\hline
\end{tabular}

(2) Test specimen size correction factor $C_{D}$

The larger the size of the specimen, the less likely it is to fatigue fracture. The correction factor of test specimen was determined by Eq. (26). In this study, the minimum diameter of the specimen is 6mm (Fig. 16). Therefore, the size correction factor is 1.0 .

$$
\left\{\begin{array}{l}
C_{D}=1.0, d_{\min }<8 m m \\
C_{D}=1.189 d-0.097,8 m m<d_{\min }<250 m m
\end{array}\right.
$$

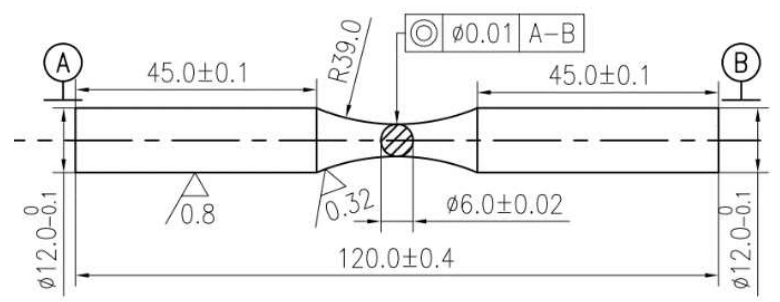

Fig. 16. Dimension drawing of test specimen

(3) Stress concentration correction factor $K_{f}$

Different surface roughness will cause different stress concentration on the surface of the specimen that will affect its fatigue life, and the formula of stress concentration correction factor was put forward by Li [62].

$$
K_{f}=1+\left(1+2 \sqrt{\lambda_{1} R_{a} / \rho}-1\right) q
$$

where $\rho$ is radius of curvature of the specimen, which is $39 \mathrm{~mm}$ (Fig. 16), $\lambda_{1}$ is the ratio of spacing to depth of micro cracks, which is $1, q$ is sensitivity coefficient, which is $0.99, R_{a}$ is the surface roughness of the specimen, which is $6.625 \mu \mathrm{m}$ measured by a white light interferometer. Therefore, the stress concentration correction factor is 1.0248 by the formula (27).

(4) Surface quality correction factor $C_{S}$

Since the fatigue crack of the test pipe mainly originates on the free surface of the test pipe, the influence of the surface quality of the test specimen on the crack needs to be considered. According to the test results of surface roughness, it can be seen from literature [62] that the surface quality correction factor can be 0.82 .

After comprehensive consideration of surface roughness, notch effect, loading type, surface quality coefficient and size coefficient, the correction formula is as follows. 


$$
S_{e}=\frac{S_{b e} C_{L} C_{S} C_{D}}{K_{f}}=0.56 S_{b e}
$$

where, $S_{b e}$ is standard stress, $S_{e}$ is corrected stress.

Finally, the corrected S-N curve of test pipe material (13Cr-L80) is obtained, as shown in Fig. 17.

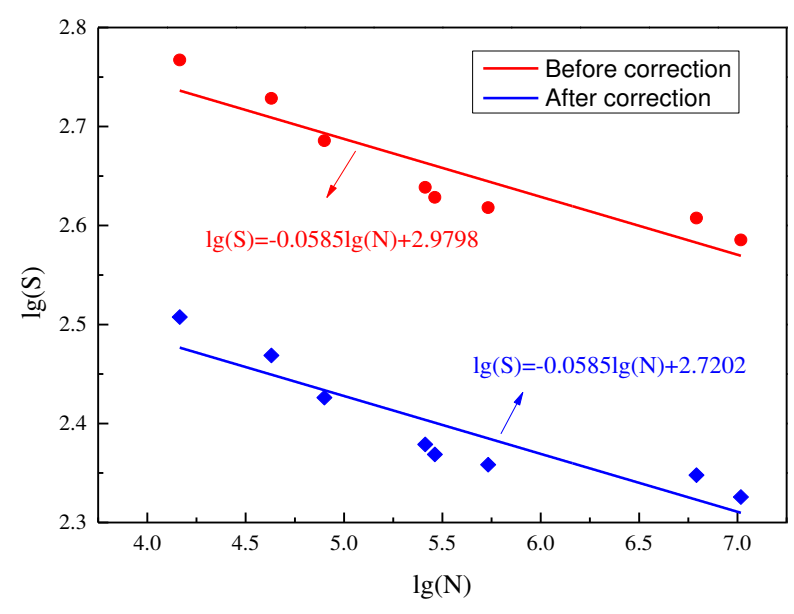

Fig. 17. S-N curve of test pipe material (13Cr-L80)

\section{Results and discussions}

According to the parameters of LS-M well in the South China Sea (as shown in Table 5) and the proposed nonlinear vibration model in Section 2, The influences of external flow velocity, internal flow velocity and top tension coefficient on the stress, fatigue life and fatigue reliability of deep-water test pipe are investigated, and the fatigue failure mechanism of deep-water test pipe is revealed.

Table 5 Calculation parameters of LS-M well

\begin{tabular}{|c|c|c|c|}
\hline Parameter & Value & Parameter & Value \\
\hline Riser and test pipe length(m) & 1000 & Time step (s) & 0.001 \\
\hline Test pipe inner diameter $(\mathrm{m})$ & 0.095 & Number of division elements & 1000 \\
\hline Test pipe outer diameter (m) & 0.1143 & Friction coefficient & 0.243 \\
\hline Riser inner diameter (m) & 0.4822 & Riser and test pipe density $\left(\mathrm{kg} / \mathrm{m}^{3}\right)$ & 7850 \\
\hline Riser outer diameter (m) & 0.533 & Seawater density $\left(\mathrm{kg} / \mathrm{m}^{3}\right)$ & 1020 \\
\hline Elastic modulus (GPa) & 210 & Drilling fluid density $\left(\mathrm{kg} / \mathrm{m}^{3}\right)$ & 1200 \\
\hline Poisson's ratio & 0.3 & Testing liquid density $\left(\mathrm{kg} / \mathrm{m}^{3}\right)$ & 1120 \\
\hline Random wave height (m) & $5 \sim 20$ & Uniform flow velocity (m/s) & $0.2 \sim 1.1$ \\
\hline Random wave period (s) & 5 & Internal flow velocity (m/s) & $5 \sim 20$ \\
\hline Rotational stiffness of the upper & & Rotational stiffness of the lower & \\
\hline flexible joint $(\mathrm{N} \cdot \mathrm{m} / \mathrm{deg})$ & 8800 & flexible joint $(\mathrm{N} \cdot \mathrm{m} / \mathrm{deg})$ & 127400 \\
\hline Calculation time (s) & 50 & Top tension coefficient & $1.1 \sim 1.7$ \\
\hline
\end{tabular}




\begin{tabular}{cccc}
\hline Hard cabin diameter $(\mathrm{m})$ & 32.31 & Heave plate spacing $(\mathrm{m})$ & 23.8 \\
Platform draft $(\mathrm{m})$ & 153.924 & Total discharge $(\mathrm{t})$ & 56401.45 \\
Depth of platform center $(\mathrm{m})$ & 90.39 & Number of heave plates & 0.0379 \\
Hard cabin length $(\mathrm{m})$ & 68.88 & Heave linear damping & 0.0186 \\
Size of Heave Plate $(\mathrm{m})$ & $32.31 \times 32.31$ & Heave quadratic damping & \\
\hline
\end{tabular}

According to the established platform heave motion model, the random wave displacement and wave spectrum are calculated. On this basis, the platform heave motion displacement is obtained (as shown in Fig. 18). It can be noted in Figs. 18(a), (b) that, random wave presents complex motion state, the amplitude of wave surface motion is less than the wave height of random wave, and the energy of random wave is concentrated between $1 \sim 1.5 \mathrm{rad} / \mathrm{s}$. It can be observed in Fig. 18(c) that, the heave amplitude of the platform is about $0.15 \mathrm{~m}$. Due to the structure of the platform, it can alleviate the influence of random waves, making its motion relatively slow and regular. The above results lay the foundation of load and displacement boundary for the response analysis of deep-water test pipe.

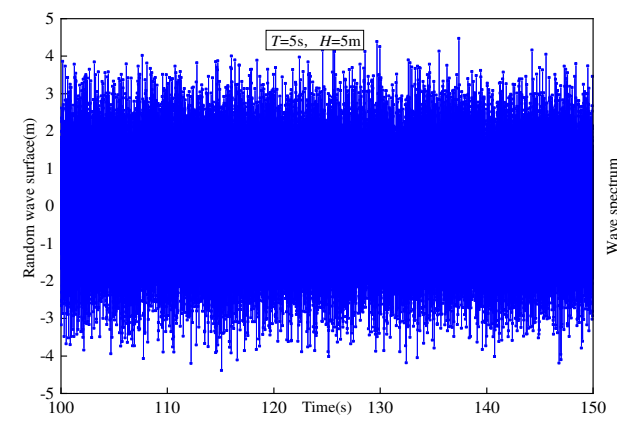

(a) Response of random wave surface

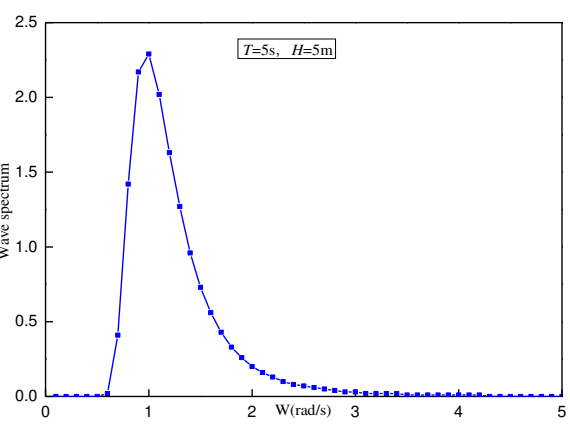

(b) Wave spectrum

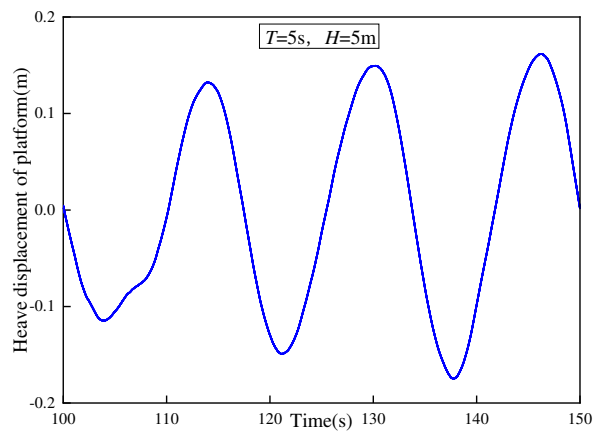

(c) Heave displacement

Fig. 18. Time history response of random wave and offshore platform

\subsection{Influence of outflow velocity}

Under the action of different outflow velocity, the riser presents different vibration states, which affects the vibration characteristics of the test pipe $[1,12,22]$. Therefore, in this study, the outflow velocity are set as $0.2,0.5,0.8$ and $1.1 \mathrm{~m} / \mathrm{s}$, and keeping the internal flow velocity, the top tension coefficient and the significant wave height unchanged, which are respectively set as $10 \mathrm{~m} / \mathrm{s}, 1.3$ and $5 \mathrm{~m}$. The other calculation parameters listed in Table 5. The curves of maximum axial stress, annual fatigue damage rate, and fatigue life corresponding to different water depths are obtained, as shown in Fig. 19. In order to display the fatigue life of test pipe conveniently, once the fatigue life is more than 60 years, it is considered as 60 years.

It can be seen from Fig. 19(a) that the maximum alternating stress of the test pipe occurs at the upper "one third" and the bottom position. The main reason is that the test pipe is affected by the contact/impact force of the outer riser, and its deformation shows the same trend as that of the riser. Meanwhile, the riser is subjected to ocean load, and its maximum stress also appear at the upper "one third" position, which was called "one third effect" by academician Zhou [1]. This is mainly because the ocean load presents a 
distribution state of large upper end and small lower end, which is similar to the shear distribution. Through the force analysis, its action point is just located at the upper one-third position. At the same time, the alternating stress at the bottom of the test pipe is also relatively large. The main reason is that during operation, the bottom of test pipe is connected with the lower marine riser packing (LMRP), which limits the axial movement of the test pipe, resulting in concentrated stress at this position. Therefore, the locations where fatigue failure of the test tube is easy to occur are mainly distributed at the upper "one third" and the bottom of test pipe (as shown in Figs. 19(b), (c) ), and the service life is about 3 years when outflow velocity is $1.1 \mathrm{~m} / \mathrm{s}$, which can meet the requirements of field short-term test (about 2 months). The field designer should pay attention to the fatigue life of the test pipe at this location.

Moreover, it can be observed from Fig. 19 that, with the increase of outflow velocity, the maximum alternating stress and annual fatigue damage rate of the test pipe increase. When the flow velocity is small (lower than $0.8 \mathrm{~m} / \mathrm{s}$ ), the increasing trend is not obvious, but when the flow velocity is large (higher than $0.8 \mathrm{~m} / \mathrm{s}$ ), the alternating stress of the test pipe varies significantly. As a result, the annual fatigue damage rate also increased significantly and the service life decreased significantly (from 8 years to 3 years). The main reason is that the increase of the outflow velocity leads to the increase of the marine load and the increase of the vibration of the riser. The alternating stress of the internal test pipe also increases through the contact/collision transmission effect. When the velocity continues to increase, the dominant frequency of the external excitation is close to that of the riser, and the riser has a resonance effect, resulting in a significant increase of the alternating stress. Finally, the alternating stress of the internal test pipe also increases significantly. Therefore, in the process of field operation, in case of severe offshore conditions (with large outflow velocity), other measures need to be taken to reduce the vibration of the test pipe (such as changing the flow velocity and top tension coefficient, etc.) to improve its fatigue life.

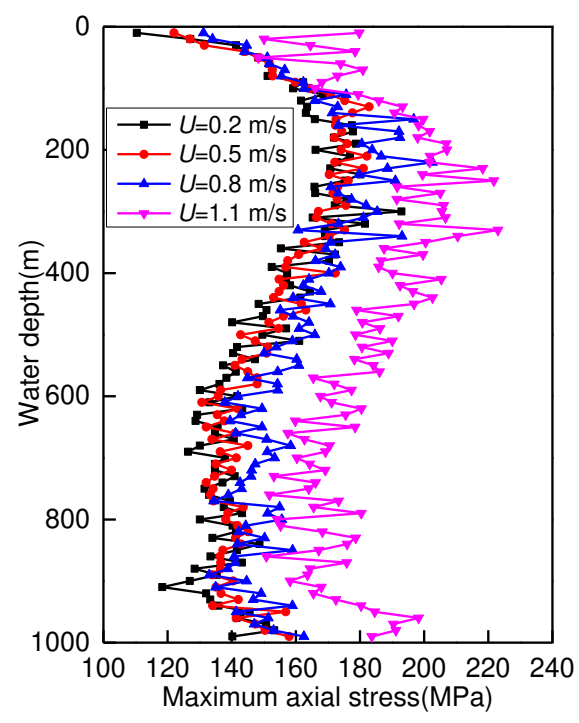

(a) Maximum axial stress

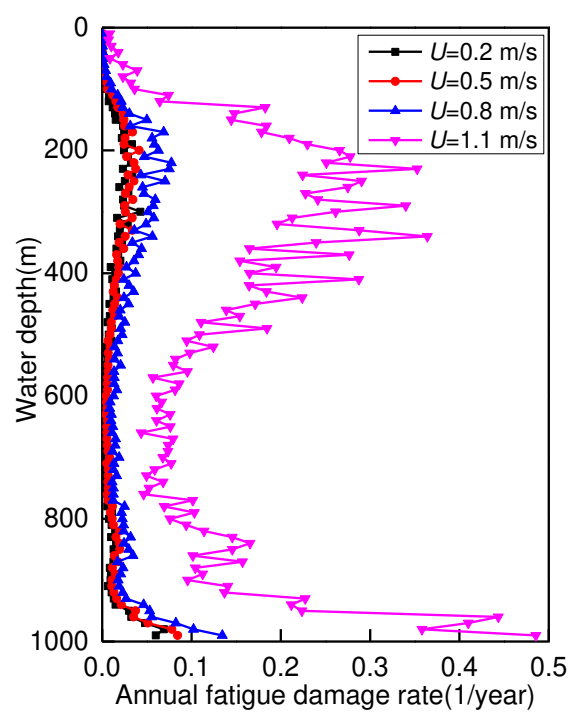

(b) Annual fatigue damage rate

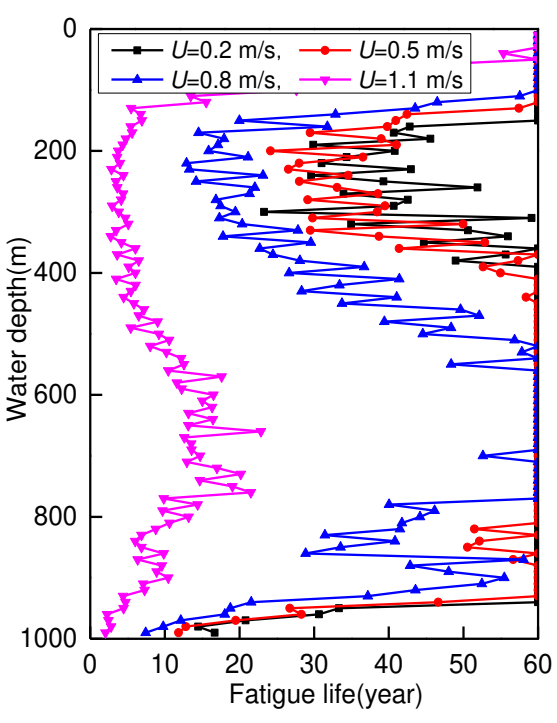

(c) Fatigue life of test pipe 


\subsection{Influence of internal flow velocity}

Deep water testing process is an important process to detect the safety of oil and gas production in the later stage. Testing the safety of the whole oil and gas production system at different oil and gas flow velocity in the test pipe can lay a foundation for the allocation of oil and gas well production in the later stage. Therefore, the internal flow velocity is an important index in the testing process. In this study, the internal velocity are set as 5,10,15 and $20 \mathrm{~m} / \mathrm{s}$, and keeping the outflow velocity, the top tension coefficient and the significant wave height unchanged, which are respectively set as $0.5 \mathrm{~m} / \mathrm{s}, 1.3$ and $5 \mathrm{~m}$. The other calculation parameters listed in Table 5. The curves of maximum axial stress, annual fatigue damage rate, and fatigue life corresponding to different water depths are obtained, as shown in Fig. 20.

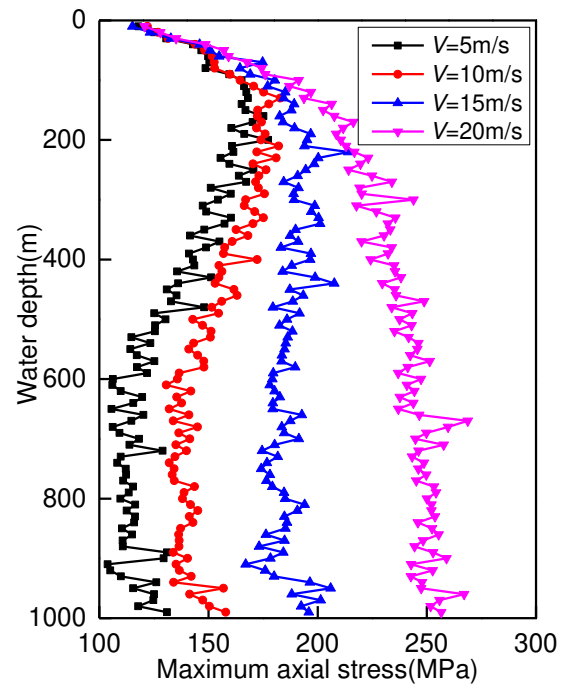

(a) Maximum axial stress

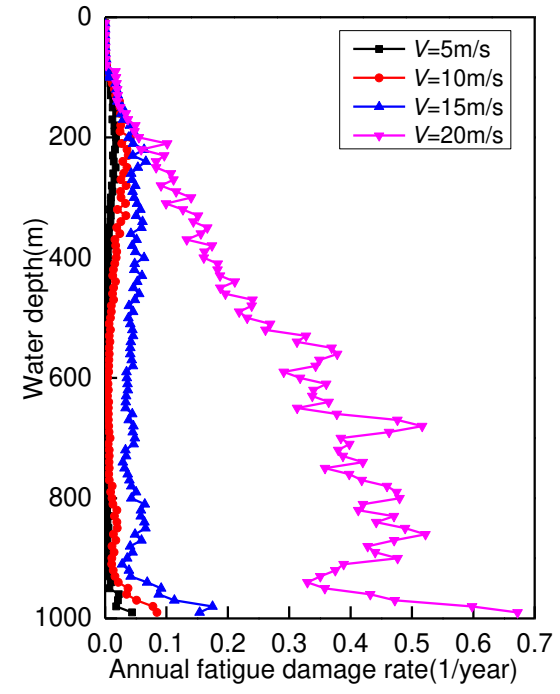

(b) Annual fatigue damage rate

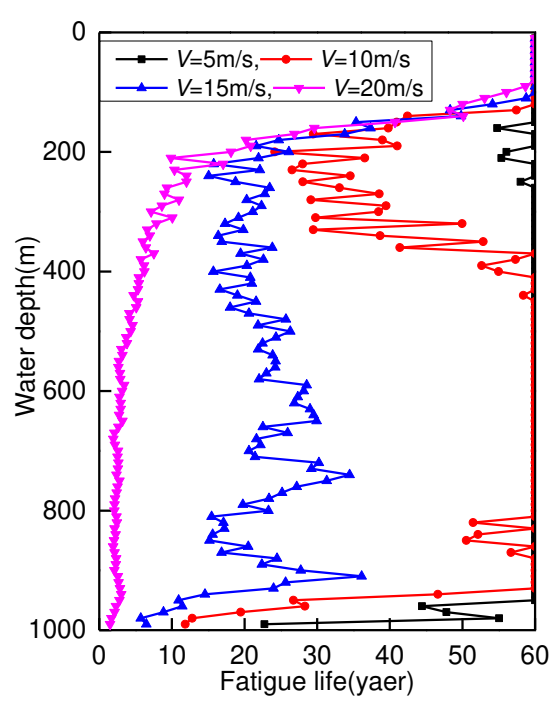

(c) Fatigue life of test pipe

Fig. 20. Fatigue damage analysis data of test pipe under different internal flow velocities

It can be noted in Fig. 20(a) that, with the increase of flow velocity in the test pipe, the alternating stress at the lower part of the test pipe increases significantly, and this increasing trend decreases in turn with the seabed to the wellhead. The main reason is that the action load of internal fluid on the test pipe mainly occurs at the position with large transverse deformation. Under the action of the test pipe selfgravity, the lower pipe string is subjected to axial compression load, and which presents a certain bending state. That results in a large flow force when the fluid in the pipe flows through these positions. Meanwhile, the upper test pipe is tensioned under its self- gravity action, and the fluid excitation is very small. Therefore, with the increase of flow velocity, the change trend of alternating stress of the test tube at the upper is very small. Finally, It can be seen that with the increase of internal flow velocity, the "one third effect" of the test pipe will decrease, and is shown "the bottom damage effect", which needs the attention of field operators.

It can be stated from Figs. 20 (b) (c) that, when the internal flow velocity is $5 \mathrm{~m} / \mathrm{s}$ and $10 \mathrm{~m} / \mathrm{s}$, the maximum annual fatigue damage rate of the test pipe occurs at the upper "one third" and the bottom position, where the fatigue life is small, about 22 years and 12 years respectively. With the increase of the internal flow velocity to $15 \mathrm{~m} / \mathrm{s}$ and $20 \mathrm{~m} / \mathrm{s}$, the annual fatigue damage rate of the test pipe at the bottom position is 
significantly greater than that at the middle and upper position, and the life decreases significantly, about 5 years and 1.5 years respectively. Therefore, during field operation, when low flow velocity is configured in the test pipe, it is necessary to focus on the fatigue damage at the upper "one third" and bottom position of the test pipe. When high flow velocity is configured in the test pipe, it is necessary to focus on the fatigue damage at the bottom position of the test pipe. The research results of this study provide a theoretical basis for field operation and parameter design.

\subsection{Influence of significant wave height}

The marine random wave mainly affects the heave motion of the platform, and then affects the vibration characteristics of the test pipe. By exploring the influence rules of the different significant wave height of marine random wave on the fatigue damage of deep-water test pipe, it can effectively guide the specific operations to be carried out on site under different sea conditions. Therefore, in this study, the significant wave height are set as 5, 10, 15 and $20 \mathrm{~m}$, and keeping the outflow velocity, the top tension coefficient and the internal velocity unchanged, which are respectively set as $0.5 \mathrm{~m} / \mathrm{s}, 1.3$ and $10 \mathrm{~m}$. The other calculation parameters listed in Table 5. The curves of maximum axial stress, annual fatigue damage rate, and fatigue life corresponding to different water depths are obtained, as shown in Fig. 21.

It can be seen from Figs. 21(a), (b), (c) that, with the increase of ocean random wave height, the alternating stress of the test pipe decreases first and then increases, but the variation amplitude is not very obvious. The main reason is that with the increase of random wave height, the heave amplitude of the platform increases, which increases the displacement of the upper end of the test tube. On the one hand, it can effectively reduce the transverse vibration of the test pipe. On the other hand, it increases the axial stress of the test pipe. When the random wave height increases slightly, the decrease trend of transverse vibration is greater than the increase trend of axial stress. The axial alternating stress of the test pipe is effectively reduced. However, when the random wave height increases again, the decreasing trend of transverse vibration is less than the increasing trend of axial stress, resulting in the increase of axial alternating stress of the test pipe. Moreover, with the increase of ocean random wave height, the annual fatigue damage rate of the test pipe first decreases and then increases, while its fatigue life first increases and then decreases. Their variation amplitude trend is similar to that of the alternating stress of the test pipe, which indicated that the increase of ocean random wave height does not affect the vibration frequency of the deep-water test pipe. The change of fatigue damage of test pipe is mainly caused by the variation of alternating stress. Therefore, during field operation, when the sea random wave height is large, it is necessary to set other parameters (such as reducing the internal flow velocity, configuring appropriate top tension coefficient, etc.) to improve the fatigue life of the test pipe. 


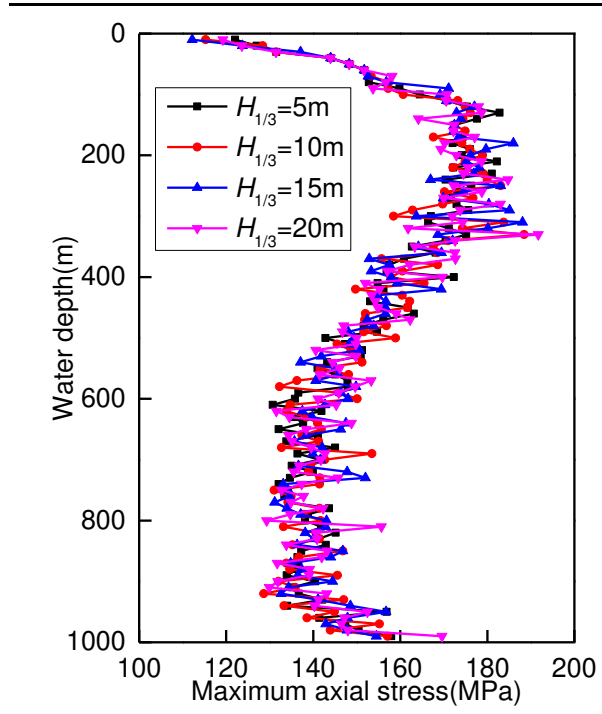

(a) Maximum axial stress

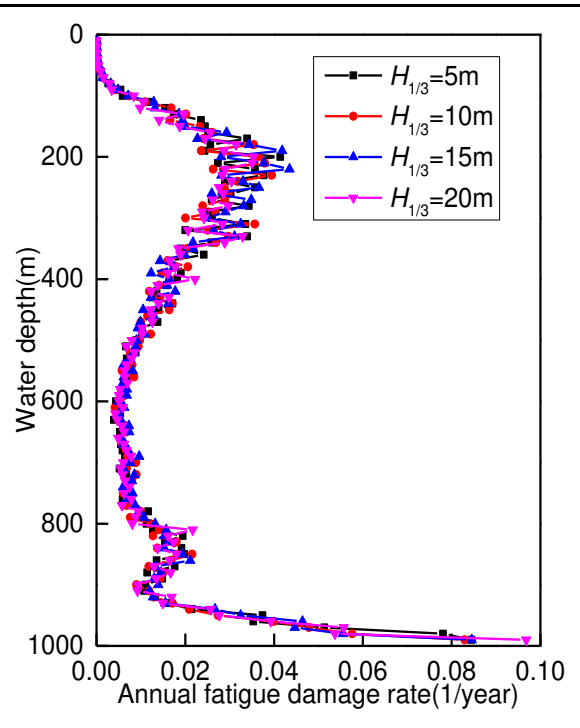

(b) Annual fatigue damage rate

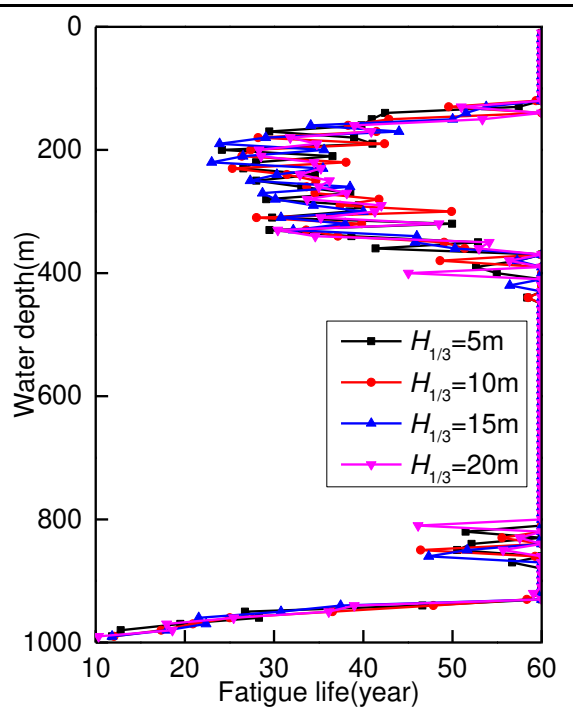

(c) Fatigue life of test pipe

Fig. 21. Fatigue damage analysis data of test pipe under different significant wave height

\subsection{Influence of top tension coefficient}

Tensioner is an important component of deep-water RTS and the only connecting component between drilling platform and submarine system. Top tension coefficient is an important mechanical parameter of tensioner, and its value determines the top tension. Different top tension will affect the vibration characteristics of deep-water test pipe, resulting in the change of its fatigue characteristics. Therefore, the influence of different top tension coefficients on fatigue damage of deep-water test pipe is explored to guide the configuration of top tension coefficient on site. In this study, the top tension coefficient are set as 1.1, 1.3, 1.5 and 1.7, and keeping the outflow velocity, the internal velocity and the significant wave height unchanged, which are respectively set as $0.5 \mathrm{~m} / \mathrm{s}, 10 \mathrm{~m} / \mathrm{s}$ and $5 \mathrm{~m}$. The other calculation parameters listed in Table 5. The curves of maximum axial stress, annual fatigue damage rate, and fatigue life corresponding to different water depths are obtained, as shown in Fig. 22.

It can be observed from Fig. 22 (a) that, with the increase of the top tension coefficient, the alternating stress of the test pipe increases, and the increasing range of the alternating stress of the test pipe along the water depth direction is similar. The main reason is that with the increase of the top tension coefficient, the top tension applied by the tensioner increases, resulting in the riser being pulled tighter. Under the action of transverse ocean load, the contact and collision effect between the riser and the test pipe is more obvious, and finally the axial alternating stress of the riser increases. Moreover, it can be observed from Fig. 22(b) that, with the increase of the top tension coefficient, the annual fatigue damage rate of the test pipe shows an increasing trend, but which increases more and more obviously, showing an uneven increasing trend. The increasing trend of annual fatigue damage rate is different from that of alternating stress of test pipe. The main reason is that with the increase of the top tension coefficient, on the one hand, the alternating stress of the test tube is increased. On the other hand, when the test tube is pulled tighter, its vibration frequency will also increase under the same external force, and this increasing trend becomes more obvious with the increase of the top tension coefficient. It can be stated from Fig. 22 (c) that, when the top tension 
coefficient changes from 1.3 to 1.5 , the fatigue life of the test pipe decreases significantly, and the minimum fatigue life is about 2.5 years. When the top tension coefficient is 1.7 , the fatigue life of the test pipe is about 0.6 years, and the test pipe is in a serious dangerous state. Therefore, during field operation, it is necessary to properly configure the top tension coefficient so that there can be a certain relaxation between the riser and the test pipe, so as to cause transverse vibration and consume some axial energy and load.

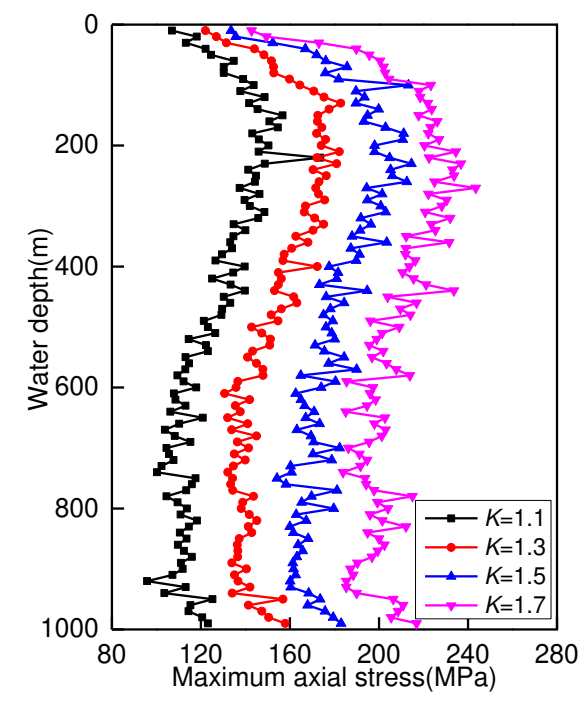

(a) Maximum axial stress

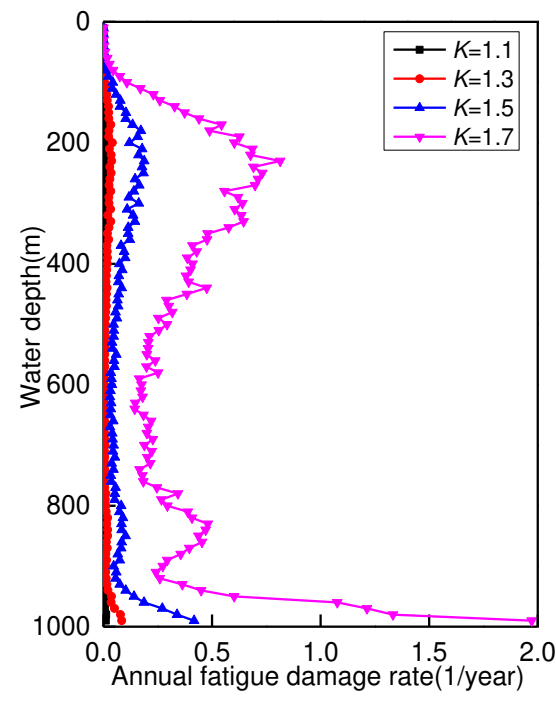

(b) Annual fatigue damage rate

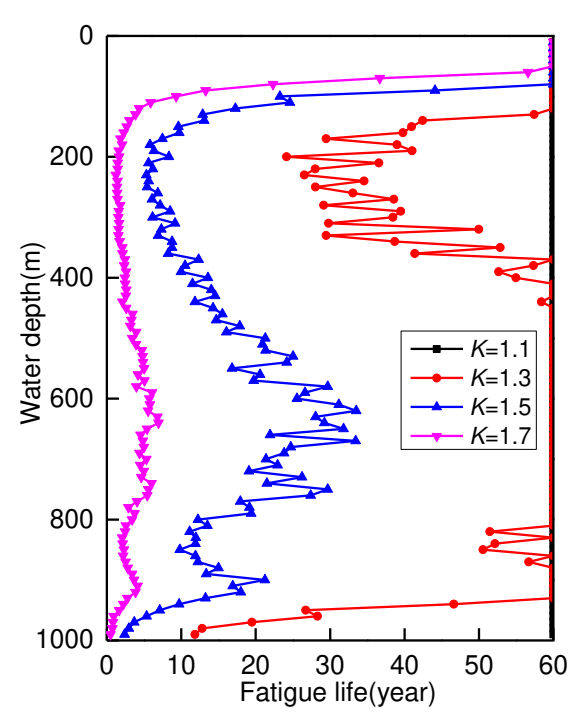

(c) Fatigue life of test pipe

Fig. 22. Fatigue damage analysis data of test pipe under different top tension coefficient

\section{Conclusions}

(1) The three-dimensional (3D) nonlinear vibration model of deep-water RTS is established using the micro-finite method, energy method and Hamilton variational principle. In the model, the vortex induced effect on riser, flow induced effect on test pipe and longitudinal/transverse coupling effect are taken into account. Based on the elastic-plastic contact collision theory, the nonlinear contact load calculation method between riser and test pipe is proposed. Due to too many nonlinear factors considered in the 3D nonlinear vibration model, only Newmark- $\beta$ method can be used for gradual integration, which will lead to the decrease of solution accuracy and the difficulty of convergence. Therefore, in this study, the incremental Newmark- $\beta$ method and Newton-Raphson method are used to solve the model simultaneously.

(2) Based on field parameters of BY-M gas well in deep-water block of South China Sea and simulation experiment area conditions, the vibration simulation test bench of the RTS is designed using geometric similarity, motion similarity, and dynamic similarity. The vibration simulation experiment of the RTS is carried out, and the vibration response data of RTS are measured. Compared with experimental measurement results, calculation results of the proposed vibration model in this study and the single tubing vibration model in our recent work, the results demonstrate that, the calculated results using the proposed model are basically consistent with the experimental data in amplitude and frequency, while the calculated results using the single tube model are quite different from the experimental data, which verifies the correctness and effectiveness of the nonlinear vibration model of the RTS.

(3) The cumulative damage theory (Miner's Law) is used to establish the fatigue life prediction method 
of test pipe combined with the stress response which was determined by the proposed 3D nonlinear model and the S-N curve of the tubing material (13Cr-L80) which was measured by fatigue test. Based on that, according to the parameters of LS-M well in the South China Sea, the influences of external flow velocity, internal flow velocity and top tension coefficient on the stress, fatigue life and fatigue reliability of deep-water test pipe are investigated.

(4) The results obtained demonstrate that, first, with the increase of outflow velocity, the maximum alternating stress, the annual fatigue damage rate increased and the service life decreased significantly. The locations where fatigue failure of the test tube is easy to occur are mainly distributed at the upper "one third" and the bottom of test pipe. Second, with the increase of internal flow velocity, the "one third effect" of the test pipe will decrease, and is shown "the bottom damage effect", which needs the attention of field operators. Third, during field operation, it is necessary to properly configure the top tension coefficient so that there can be a certain relaxation between the riser and the test pipe, so as to cause transverse vibration and consume some axial energy and load.

\section{Acknowledgments}

This work was partially supported by the National Natural Science Foundation of China (Grant No. 52105125 and No. 51875489), the China Postdoctoral Science Foundation (Grant No. 2021TQ0273), Sichuan Province Youth Science and Technology Innovation Team (Grant No. 2019JDTD0017).

\section{Conflicts of interest}

The authors declare that there are no conflicts of interest regarding the publication of this paper.

\section{Data Availability Statements}

The authors declare that the data supporting the findings of this study are available within the article.

\section{Nomenclature}

\begin{tabular}{|c|c|c|c|}
\hline$m^{*}$ & mass ratio of riser & $v_{i}, i=x, y, z$ & ement components of riser, $\mathrm{m}$ \\
\hline E & elastic modulus of the RTS, Pa & $A_{v}$ & $f$ the riser, $\mathrm{m}^{2}$ \\
\hline$v_{i}^{\prime}, i=x, y, z$ & $\begin{array}{l}\text { first-order derivative of riser } \\
\text { displacements versus } z\end{array}$ & $v_{i}^{\prime \prime}, i=x, y, z$ & $\begin{array}{l}\text { second derivative of riser } \\
\text { displacements versus } z\end{array}$ \\
\hline$\dot{v}_{i}, i=x, y, z$ & $\begin{array}{l}\text { first-order derivative of riser } \\
\text { displacements versus time }\end{array}$ & $I_{v}$ & $\begin{array}{l}\text { polar moment of inertia of the riser, } \\
\mathrm{m}^{4}\end{array}$ \\
\hline$\rho_{v}$ & density of the riser, $\mathrm{kg} / \mathrm{m}^{3}$ & $m_{v}$ & mass of the per unit length riser, $\mathrm{kg}$ \\
\hline$F_{x}(z, t)$ & $\begin{array}{l}\text { contact/impact force of riser- } \\
\text { test pipe in } x \text {-directions, } \mathrm{N}\end{array}$ & $F_{y}(z, t)$ & $\begin{array}{l}\text { contact/impact force of riser-test pipe } \\
\text { in } y \text {-directions, } \mathrm{N}\end{array}$ \\
\hline
\end{tabular}




\begin{tabular}{|c|c|c|c|}
\hline$F_{z}(z, t)$ & $\begin{array}{l}\text { friction force of riser-test pipe in } \\
z \text {-directions, } \mathrm{N}\end{array}$ & $c_{v}\left(=2 m_{v} \omega_{v} \zeta\right)$ & structural damping coefficient of riser \\
\hline$F_{L}(z, t)$ & lateral lift in the $\mathrm{CF}$ direction, $\mathrm{N}$ & $F_{D}(z, t)$ & drag force in the IL direction, $\mathrm{N}$ \\
\hline$\omega_{v}$ & $\begin{array}{l}\text { natural angular frequency of } \\
\text { riser }\end{array}$ & $w_{g}$ & $\begin{array}{l}\text { buoyant weight of riser per unit } \\
\text { length, } \mathrm{N}\end{array}$ \\
\hline$L_{v}$ & length of riser, $m$ & $\rho_{w}$ & density of the sea-water, $\mathrm{kg} / \mathrm{m}^{3}$ \\
\hline$D_{o}$ & riser outer diameter, $\mathrm{m}$ & $A_{s}$ & $\begin{array}{l}\text { cross-sectional area of the test pipe, } \\
\mathrm{m}^{2}\end{array}$ \\
\hline$m_{i}$ & $\begin{array}{l}\text { the mass of the gas per unit } \\
\text { length }(\mathrm{kg})\end{array}$ & $m_{s}$ & $\begin{array}{l}\text { mass of the per unit length test pipe, } \\
\mathrm{kg}\end{array}$ \\
\hline$S_{i}, i=x, y, z$ & $\begin{array}{l}\text { displacement components of the } \\
\text { test pipe, } m\end{array}$ & $S_{i}^{\prime}, i=x, y, z$ & $\begin{array}{l}\text { first-order derivative of test pipe } \\
\text { displacements versus } z\end{array}$ \\
\hline$S_{i}^{\prime \prime}, i=x, y, z$ & $\begin{array}{l}\text { second derivative of test pipe } \\
\text { displacements versus } z\end{array}$ & $\dot{S}_{i}, i=x, y, z$ & $\begin{array}{l}\text { first-order derivative of test pipe } \\
\text { displacements versus time }\end{array}$ \\
\hline$f_{x}(z, t)$ & $\begin{array}{l}\text { high-speed fluid impact load in } \\
\text { test pipe in } x \text {-direction, } \mathrm{N}\end{array}$ & $f_{y}(z, t)$ & $\begin{array}{l}\text { high-speed fluid impact load in test } \\
\text { pipe in } y \text {-direction, } \mathrm{N}\end{array}$ \\
\hline$f_{z}(z, t)$ & $\begin{array}{l}\text { high-speed fluid impact load in } \\
\text { test pipe in } z \text {-direction, } \mathrm{N}\end{array}$ & $c_{s}\left(=2 m_{s} \omega_{s} \zeta\right)$ & $\begin{array}{l}\text { structural damping coefficient of test } \\
\text { pipe }\end{array}$ \\
\hline$\omega_{s}$ & $\begin{array}{l}\text { natural angular frequency of test } \\
\text { pipe }\end{array}$ & $v_{i}, i=x, y, z$ & $\begin{array}{l}\text { absolute velocities of the internal } \\
\text { high-speed fluid }(\mathrm{m} / \mathrm{s})\end{array}$ \\
\hline$w_{s}\left(=m_{s} g\right)$ & $\begin{array}{l}\text { weight of test pipe per unit } \\
\text { length, } \mathrm{N}\end{array}$ & $V$ & $\begin{array}{l}\text { fluid flow velocity in the test pipe, } \\
\mathrm{m} / \mathrm{s}\end{array}$ \\
\hline$V_{r}$ & $\begin{array}{l}\text { relative velocity between the } \\
\text { fluid and the riser, } \mathrm{m} / \mathrm{s}\end{array}$ & $\bar{C}_{d}$ & coefficient of steady-state drag force \\
\hline$U_{c}$ & outflow velocity of the riser, $\mathrm{m} / \mathrm{s}$ & $\bar{C}_{l}$ & coefficient of steady lift force \\
\hline$F_{D}{ }^{\prime}, C_{D}$ & $\begin{array}{l}\text { component forces of the } \\
\text { fluctuating drag force and } \\
\text { corresponding coefficient }\end{array}$ & $F_{L}^{\prime}, C_{L}$ & $\begin{array}{l}\text { fluctuating lift force and } \\
\text { corresponding coefficient }\end{array}$ \\
\hline$q_{i}, i=x, y$ & $\begin{array}{l}\text { dimensionless wake oscillator } \\
\text { variables in IL and } \mathrm{CF} \\
\text { directions }\end{array}$ & $z_{i}, i=1,2$ & $\begin{array}{l}\text { radial distance from the contact point } \\
\text { of pipe to the inner wall of riser, } m\end{array}$ \\
\hline$S_{t}$ & Strouhal number & $\omega_{s}^{\prime}$ & vortex shedding frequency \\
\hline$R_{i}, i=1,2$ & radius of riser and test pipe, $\mathrm{m}$ & $r$ & $\begin{array}{l}\text { horizontal distance from the contact } \\
\text { point to the test pipe axis, } m\end{array}$ \\
\hline$\omega_{i}, i=1,2$ & axial displacements of the riser & $F$ & contact load of riser-test pipe, $\mathrm{N}$ \\
\hline
\end{tabular}


and test pipe, $\mathrm{m}$

\begin{tabular}{|c|c|c|c|}
\hline$E_{i}, i=1,2$ & $\begin{array}{l}\text { elastic modulus of the riser and } \\
\text { test pipe material, } \mathrm{Pa}\end{array}$ & $\mu_{i}, i=1,2$ & $\begin{array}{l}\text { Poisson's ratio of riser and test pipe } \\
\text { material }\end{array}$ \\
\hline$q(x)$ & $\begin{array}{l}\text { uniform load distribution for } \\
\text { riser and test pipe, } \mathrm{N}\end{array}$ & $\xi$ & $\begin{array}{l}\text { friction coefficient between the riser } \\
\text { and test pipe }\end{array}$ \\
\hline$\rho_{i}$ & $\begin{array}{l}\text { density of gas in the test pipe, } \\
\mathrm{kg} / \mathrm{m}^{3}\end{array}$ & $A_{i}$ & $\begin{array}{l}\text { cross-sectional area of the wellbore, } \\
\mathrm{m}^{2}\end{array}$ \\
\hline$\alpha(t)$ & $\begin{array}{l}\text { deflection angles of test pipe in } \\
x \text { - direction, rad }\end{array}$ & $\varphi(t)$ & $\begin{array}{l}\text { deflection angles of test pipe in } y \text { - } \\
\text { direction, rad }\end{array}$ \\
\hline$\alpha(s)$ & inclination angle, rad & $\varphi(s)$ & azimuth, rad \\
\hline$K_{\mathrm{U}}$ & $\begin{array}{l}\text { rotational stiffness of the upper } \\
\text { flexible joint }\end{array}$ & $K_{\mathrm{L}}$ & rotation stiffness of the BOP \\
\hline$u_{\text {boat }}(t)$ & $\begin{array}{l}\text { heave displacement of the } \\
\text { platform, } m\end{array}$ & $B_{i}, i=1,2$ & $\begin{array}{l}\text { heave radiation and heave viscous } \\
\text { damping }\end{array}$ \\
\hline$m_{\mathrm{p}}$ & mass of platform, $\mathrm{kg}$ & $A_{w}$ & area of the platform at sea level, $\mathrm{m}^{2}$ \\
\hline$\eta(t)$ & $\begin{array}{l}\text { surface displacement of random } \\
\text { wave, } m\end{array}$ & $\bar{F}_{z}$ & $\begin{array}{l}\text { random heave wave exciting force on } \\
\text { platform, } \mathrm{N}\end{array}$ \\
\hline$\hat{\omega}_{i}$ & $\begin{array}{l}\text { circular frequency of the } i \text { th } \\
\text { harmonic, } \mathrm{Hz}\end{array}$ & $\varepsilon_{i}$ & $\begin{array}{l}\text { initial phase of the } i \text { th harmonic } \\
\text { component, rad }\end{array}$ \\
\hline$a_{i}$ & $\begin{array}{l}\text { amplitude of the } i \text { th harmonic } \\
\text { component, } \mathrm{m}\end{array}$ & $\Delta \omega$ & frequency step \\
\hline$S(\omega)$ & random wave spectrum & $f$ & Frequency, $\mathrm{Hz}$ \\
\hline$\omega$ & circular frequency, $\mathrm{Hz}$ & $H_{1 / 3}$ & significant wave height, $\mathrm{m}$ \\
\hline$T_{1 / 3}$ & significant period of the wave, $\mathrm{s}$ & $f_{p}$ & peak frequency of the wave, $\mathrm{Hz}$ \\
\hline$T_{p}$ & peak period of the wave, $s$ & $\gamma$ & peak parameter \\
\hline$\sigma$ & peak shape coefficient & $R$ & platform radius, $\mathrm{m}$ \\
\hline$F_{p}(t)$ & $\begin{array}{l}\text { exciting force of the random } \\
\text { wave on the heave plate, } \mathrm{N}\end{array}$ & $F_{s}(t)$ & $\begin{array}{l}\text { exciting force of the random wave on } \\
\text { the platform body, } \mathrm{N}\end{array}$ \\
\hline$J_{1}(\cdot)$ & $\begin{array}{l}\text { first order Bessel function of } \\
\text { first kind }\end{array}$ & $d$ & draft of platform, $m$ \\
\hline$z_{\text {plate }}$ & depth of heave plate, $m$ & $B_{\text {plate }}$ & width of heave plate, $\mathrm{m}$ \\
\hline d & displacement vector of riser unit & $\overline{\mathbf{d}}$ & displacement vector of test pipe unit \\
\hline $\boldsymbol{\varphi}_{i}, i=x, y, z$ & $\begin{array}{l}\text { vibration shape function of riser } \\
\text { and test pipe unit }\end{array}$ & $\mathbf{D}$ & matrix of overall displacement \\
\hline
\end{tabular}




\begin{tabular}{|c|c|c|c|}
\hline $\mathbf{F}(t)$ & load column vector & $\mathbf{K}(t)$ & matrices of the overall stiffness \\
\hline $\mathbf{M}(t)$ & matrices of the overall mass & $\mathbf{C}(t)$ & matrices of the overall damping \\
\hline$\rho_{\mathrm{p}}$ & density of the actual RTS, $\mathrm{kg} / \mathrm{m}^{3}$ & $E_{\mathrm{p}}$ & elastic modulus of the actual RTS, Pa \\
\hline$\rho_{\mathrm{m}}$ & $\begin{array}{l}\text { density of the RTS in the } \\
\text { simulation experiment }\end{array}$ & $E_{\mathrm{m}}$ & $\begin{array}{l}\text { elastic modulus of the RTS in the } \\
\text { simulation experiment }\end{array}$ \\
\hline$\lambda$ & radial similarity ratio & $T_{f}$ & service life of test pipe, year \\
\hline$C_{L}$ & load type correction factor & $C_{D}$ & test specimen size correction factor \\
\hline$K_{f}$ & $\begin{array}{l}\text { stress concentration correction } \\
\text { factor }\end{array}$ & $R_{a}$ & $\begin{array}{l}\text { surface roughness of the specimen, } \\
\mu \mathrm{m}\end{array}$ \\
\hline$C_{S}$ & surface quality correction factor & $S_{b e}$ & standard stress, $\mathrm{Pa}$ \\
\hline$S_{e}$ & corrected stress, Pa & $D^{\prime}$ & total fatigue damage \\
\hline
\end{tabular}

\section{Abbreviation}

$\begin{array}{ll}\text { RTS } & \text { riser-test pipe system } \\ \text { VIV } & \text { vortex-induced vibration } \\ \text { CF } & \text { crossflow } \\ \text { BOP } & \text { blowout preventer } \\ \text { LMRP } & \text { lower marine riser packing }\end{array}$

$3 \mathrm{D}$

CFD

IL

RMS three-dimensional

computational fluid dynamics

inline

root mean square

\section{References}

[1] Zhou, S., Liu, Q., Jiang, W., Mao, L., Yang, X., Liu, Z., Wang, G., Huang, X., Shi, X., 2013. The discovery of “one third effect" for deep water drilling riser: based on the theoretical and experimental study of the deformation characteristics of deep water drilling riser by ocean currents. China Offshore Oil and Gas, 25(6):1-7.

[2] Sarpkaya, T., 1979. Vortex-induced oscillations-a selective review. Journal of Applied Mechanics, 46(2):241-258.

[3] Bearman, P.W., 2003. Vortex shedding from oscillating bluff bodies. Annual Review of Fluid Mechanics, 16(1):195222.

[4] Dahl, J.M., Hover, F.S., Triantafyllou, M.S., Oakley, O.H., 2010. Dual resonance in vortex-induced vibrations at subcritical and supercritical reynolds numbers. Journal of Fluid Mechanics, 643(3):395-424.

[5] Facchinetti, M.L., Langre, E.D., Biolley, F., 2004. Coupling of structure and wake oscillators in vortex-induced vibrations. Journal of Fluids \& Structures, 19(2):123-140.

[6] Vandiver, J.K., Jaiswal, V., Jhingran, V., 2009. Insights on vortex-induced, traveling waves on long risers. Journal of Fluids \& Structures, 25(4):641-653.

[7] Huera-Huarte, F.J., Bangash, Z.A., González, L.M., 2014. Towing tank experiments on the vortex-induced vibrations of low mass ratio long flexible cylinders. Journal of Fluids \& Structures, 48:81-92.

[8] Gao, Y., Fu, S., Wang, J., Song, L., Chen, Y., 2015. Experimental study of the effects of surface roughness on the vortex-induced vibration response of a flexible cylinder. Ocean Engineering 103(1):40-54

[9] Bourguet, R., Karniadakis, G.E., Triantafyllou, M.S., 2011. Vortex-induced vibrations of a long flexible cylinder in shear flow. Journal of Fluid Mechanics, 677(677):342-382. 
[10] Bourguet, R., Karniadakis, G.E., Triantafyllou, M.S., 2013. Distributed lock-in drives broadband vortex-induced vibrations of a long flexible cylinder in shear flow. Journal of Fluid Mechanics, 717(1):361-375.

[11] Mao, L., Zeng, S., Liu, Q., 2019. Dynamic mechanical behavior analysis of deep water drilling riser under hard hangoff evacuation conditions. Ocean Engineering, 183, 318-331.

[12] Mao, L., Zeng, S., Liu, Q., Wang, G., He, Y., 2020. Dynamical mechanics behavior and safety analysis of deep water riser considering the normal drilling condition and hang-off condition. Ocean Engineering, 199, 106996.

[13] Mathelin, L., Langre, E., 2005. Vortex-induced vibrations and waves under shear flow with a wake oscillator model. European Journal of Mechanics - B/Fluids, 24(4):478-490.

[14] Khalak, A., Williamson, C.H.K., 1999. Motions, forces and mode transitions in vortex-induced vibrations at low mass-damping. Journal of Fluids \& Structures, 13(7-8):813-851.

[15] Xu, J., Wang, D., Huang, H., Duan, M., Gu, J., An, C., 2017. A vortex-induced vibration model for the fatigue analysis of a marine drilling riser. Ships \& Offshore Structures, 12(sup1) S280-S287

[16] He, F., Dai, H., Huang, Z., Wang, L., 2017. Nonlinear dynamics of a fluid-conveying pipe under the combined action of cross-flow and top-end excitations. Applied Ocean Research, 62:199-209

[17] Jauvtis, N., Williamson, C.H.K., 2003. Vortex-induced vibration of a cylinder with two degrees of freedom. Journal of Fluids \& Structures, 17(7):1035-1042

[18] Gu, J., Yang, C., Zhu, X.Y., Wu, J., 2016. Influences of mass ratio on vortex induced vibration characteristics of a circular cylinder. Journal of Vibration \& Shock, 35(4):134-140.

[19] Martins, F.A.C., Avila, J.P.J., 2019. Effects of the Reynolds number and structural damping on vortex-induced vibrations of elastically-mounted rigid cylinder. International Journal of Mechanical Sciences, 156, 235-249.

[20] Gao, G., Cui, Y., Qiu X., Shu, Q., 2019. Parameter influencing analysis of vortex-induced vibration response of deep sea top tensioned riser. Shipbuilding Engineering, 41(2): 101-107.

[21] Liu, J., Zhao, H., Liu, Q., He, Y., Wang, G., Wang, C., 2018. Dynamic behavior of a deepwater hard suspension riser under emergency evacuation conditions. Ocean Engineering, 150, 138-151.

[22] Liu, J., Guo, X., Liu, Q., Wang, G., He, Y., Li, J., 2019. Vortex induced vibration response characteristics of marine riser considering the in-line and cross-flow coupling effect. Acta Petrolei Sinica, 40(10):1270-1280.

[23] Aitken, J., 1878. An account of some experiments on rigidity produced by centrifugal force. Philosophical Magazine. 5, 81-105.

[24] Shilling, R., Lou, Y.K., 1980. An experimental study on the dynamic response of a vertical cantilever pipe on the dynamic response of a vertical cantilever pipe conveying fluid. Journal of Energy Resources Technology. $102,129-135$.

[25] Deng, Y.Z., 2006. Vibration mechanism analysis and fatigue life prediction of tubing string in high yield gas well. Southwest Petroleum University.

[26] Paidoussis, M.P., Luu, T.P., Prabhakar, S., 2008. Dynamics of a long tubular cantilever conveying fluid downwards, which then flows upwards around the cantilever as a confined annular flow. Journal of Fluids and Structures. 24(1), 111-128.

[27] Ju, Q.L., Tong, S.K., 2014. Analysis of vertical vibration characteristics of completion string of high yield gas well. Drilling and Production Technology. 37 (02), 79-82+119-120.

[28] Zhang, Q., Miska, S., 2003. Lateral vibration of nonrotating drill pipe conveying fluid in vertical holes. Asme/jsme Joint Fluids Summer Engineering Conference, pp. 109-120. 
[29] Bagdatli, S., 2015. Non-linear transverse vibrations of tensioned nanobeams using nonlocal beam theory. Structural Engineering and Mechanics. 55(2), 281-298.

[30] Dai, H.L., Abdelkefi, A., Wang, L., 2014. Modeling and nonlinear dynamics of fluid-conveying risers under hybrid excitations. International Journal of Engineering Science. 81, 1-14.

[31] Yu, K.Q., 2017. Fluid solid coupling vibration analysis and safety evaluation of completion string of high production gas well. Xi'an University of petroleum.

[32] Xing, Y.F., Liang, K., 2015. Nonlinear vibration analysis of longitudinal-transverse coupled beam. Journal of Beijing University of Aeronautics and Astronautics. 41(08), 1359-1366.

[33] Li, Z.F., 2016. Research advances and debates on tubular mechanics in oil and gas well. Acta petrolei sinica. 37(4), 531-556.

[34] Liu, J., Zhao, H., Yang, S., Liu, Q., Wang, G., 2018. Nonlinear dynamic characteristic analysis of a landing string in deepwater riserless drilling. Shock and Vibration. Article ID: 8191526.

[35] Wang, W.M., Xiong, M.H., Chen, Q.R., Chen, Y.C., Zhang, S.M., Yang, D.F., 2015. Simulation analysis of load transfer in deep sea pipe-in-pipe vertical system. Oil Field Equipment. 3, 1-5.

[36] Li, C.N., 2017. Buckling of concentric string pipe-in-pipe. SPE-187445-MS.

[37] Liu, K., Chen, G.M., Chang, Y.J., Zhu, B.R., Liu, X.Q., 2016. Nonlinear dynamic analysis and fatigue damage assessment for a deepwater test string subjected to random loads. Petroleum Science. 13(1), 126-134.

[38] Yang, M.J., Li, H.T., Jiang, R., Xiao, K.W., Xie, J., 2016. Research on vibration damage of completion string in Yolotan high-yield gas well. Journal of Southwest Petroleum University (Science \& Technology Edition). 38(01), 158-163.

[39] Liu, J., Guo, X., Wang, G., Liu, Q., Fang, D., Huang, L., Mao, J., 2020. Bi-nonlinear vibration model of tubing string in oil\&gas well and its experimental verification. Applied Mathematical Modelling. 81, 50-69.

[40] Guo, X., Liu, J., Wang, G., Dai, L., Fang, D., Huang, L., 2021. Nonlinear flow-induced vibration response characteristics of a tubing string in HPHT oil\&gas well. Applied Ocean Research. 102468.

[41] Drumond, G.P., Pasqualino, I.P., Pinheiro, B.C., Estefen, S.F., 2018. Pipelines, risers and umbilicals failures: A literature review. Ocean Engineering.148, 412-425.

[42] Lekkala, M.R., Mohamed, L., Hafiz, M.F.U., Kim D.K., 2020. A practical technique for hydrodynamic coefficients modification in SHEAR7 for fatigue assessment of riser buoyancy modules under vortex -induced vibration. Ocean Engineering.217, 107760.

[43] Chen, S.S., Wang, H.X., Liu, Y.X., Lan, W.J., Lv, X.X., Sun, B.Y., 2019. Root cause analysis of tubing and casing failures in low-temperature carbon dioxide injection well. Engineering Failure Analysis. 104, 873-886.

[44] Hejazi, R., Grime, A., Randolph, M., Efthymiou, M., 2021. A Bayesian machine learning approach to rapidly quantifying the fatigue probability of failure for steel catenary risers. Ocean Engineering. 235, 109353.

[45] Chen, R.F., Low, Y.M., 2021. Efficient long-term fatigue analysis of deepwater risers in the time domain including wave directionality. Marine Structures. 78, 103002.

[46] Zhao, H.L, 2019. Study on the mechanism of vortex -induced vibration of marine riser under the influence of nonlinear factors[D]. Chengdu: Southwest Petroleum University.

[47] Xu, B.Y., 1984. Elastoplastic mechanics and its application. Beijing, China Machine Press.

[48] Violette, R., Langre-e, DE, Szydlowski, J., 2007. Computation of vortex-induced vibrations of long structures using a wake oscillator model: Comparison with DNS and experiments. Comput Struct. 85(11-14): 1134-1141.

[49] Wen, S.T., Huang, P., Tian, Y., Ma, L.R., 2018. Principles of tribology fifth edition. Beijing, Tsinghua university press.

[50] Guo, X., Liu, J., Dai, L., Liu, Q., Fang, D., Wei, A., Wang, J., 2021. Friction-wear failure mechanism of tubing 
strings used in high-pressure, high-temperature and high-yield gas wells. Wear. Mar., 468-469.

[51] Luo, T., 2017. Fluid Mechanics. Beijing: Machinery Industry Press.

[52] Liu, Q.Y., He, Y.F., 2013. Mechanical behavior of injection string in deep well and its application. Beijing: Science Press.

[53] Mao, L., Liu, Q., Zhou, S., Wang, G., Fu, Q., 2016. Deep water drilling riser mechanical behavior analysis considering actual riser string configuration. Journal of Natural Gas Science and Engineering. 33: 240-254.

[54] Shen, W.J., 2012. Study on the Nonlinear Stochastic Dynamic Response Characteristics of a Truss Spar. Tianjin: Tianjin University.

[55] Longuet-Higgins, M.S., 1963. The effect of non-linearities on statistical distributions in the theory of sea waves. Journal of Fluid Mechanics, 17(03): 459-480.

[56] Guo X.Q., Li, X., Liu J., Dai L.M., Huang L., Wei A.C., Zhang X.H. 2021. Fatigue failure mechanism of 3D tubing strings used in high-pressure, high-temperature and high-yield curved gas wells. Engineering Failure Analysis. 128: 105536.

[57] Tang, Y.G., Pan, Y.R., Zhang, J., Wang, B., 2016. Experiment on vortex induced vibration of deep sea risers considering parametric excitations. Journal of Tianjin University, 49(1), 58-64.

[58] Li, Z.F., Wang, C.J., Tian, W.C., Xie, J., 2017. Three principles of drill string mechanics and qualitative simulation experiments. Acta Petrolei Sinica. 38(2), 227-223.

[59] Cardarelli, F., 2018. Materials handbook. A concise desktop reference $3^{\text {rd }}$ edition. Springer.

[60] Zhang, T., 2018. Review on research and development of fatigue life analysis methods. Agricultural equipment and vehicle engineering. 56 (11), 91-93.

[61] Li, B., 2015. Application of rain flow counting method in structural fatigue damage calculation. Science and Technology Horizon. (16), $190+244$.

[62] Li, Y.L., 2011. Theory and practice of fatigue test analysis. Trans. Zhang Ranzhi. Beijing, National Defense Industry Press. 102-110. 